\title{
A structured control model for the thermo-magneto-hydrodynamics of plasmas in tokamaks
}

Ngoc Minh Trang VU ${ }^{a}$, Laurent LEFÈVRE ${ }^{b \star 1}$ and Bernhard MASCHKE ${ }^{c}$

${ }^{a}$ CEA, IRFM, F-13108 Saint-Paul-Lez-Durance, France

${ }^{b}$ Univ. Grenoble Alpes, LCIS, F-26902, France

${ }^{c}$ Laboratoire d'Automatique et Génie des Procédés, Université Claude Bernard Lyon 1, Lyon, France

Email: ngoc-minh-trang.vu@cea.fr; laurent.lefevre@lcis.grenoble-inp.fr; maschke@lagep.univ-lyon1.fr

\section{Introduction}

A tokamak is a facility constructed with the shape of a torus (or doughnut) in which a plasma is magnetically confined and heated in order to produce nuclear fusion reactions (see Fig. 1 for a schematic view and the classical Wesson's monograph [54] for a large comprehensive reference textbook). The ITER (International Thermonuclear Experimental Reactor) project is currently building the world's largest experimental tokamak nuclear fusion reactor. However many challenges remain to prove the scientific feasibility of this goal and then to move towards a fully functional plant. Some of these are control problems such as $[39,53,2]$ the vertical stabilization of the plasma, controlling the overall shape of the plasma, controlling the magneto-hydrodynamic (MHD) instabilities or controlling the current, temperature and pressure density profiles.

There exist many kinds of models for the plasma dynamics in tokamaks including the 1D resistive diffusion equation for the poloidal flux [6], Hamiltonian formulation for the microscopic kinetic equations [29, 26] or macroscopic fluid-like description derived from these microscopic equations [46].

Up to now, the so-called $1 \mathrm{D}$ resistive diffusion model for the poloidal flux (see [6, Chap.6] for model derivation and details) has been extensively used as a "control model" for many control designs related to the plasma total current or current profile in the tokamak. In this model the plasma is assumed to have reached the hydrodynamic equilibrium profile and displacement currents are 


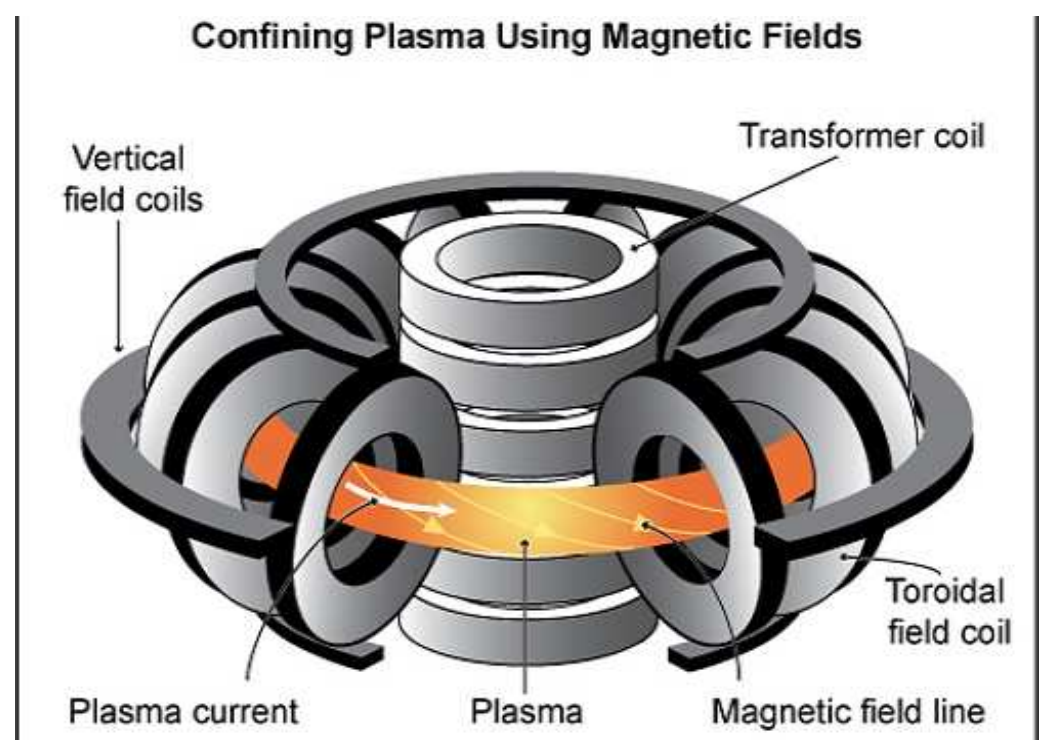

Figure 1: Schematic view of a tokamak with the electrical solenoids: the magnetic field generated by the three magnets makes the plasma gas ions following helicoidal trajectories along the torus

neglected in the Maxwell equations. The model then reduces to a $1 \mathrm{D}$ diffusionlike parabolic equation for the radial profile of the plasma poloidal flux (or current density). Readers could refer to [55] for investigations on this model for control purposes or to [38] for application to model-based predictive control. A similar model has been used to solve the current profile optimal tracking problem [37] or to design robust controller for the poloidal magnetic flux profile [36]. Feedback control using Lyapunov approach in [1], or sliding mode in [18] are also proposed.

However, this 1D resistive diffusion model makes very restrictive assumptions on the plasma resistivity (which in fact depends strongly and non-linearly of the temperature) and neglects some nonlinear MHD couplings which produces among others the bootstrap current ${ }^{2}$. The next objective for plasma high confinement requires now a better understanding and exploitation (for control purposes) of TMHD interdomain couplings. Moreover, a study of the complete TMHD model, including mass and entropy balance equations is necessary to consider the burn control problem (i.e. control of the plasma with the fusion reaction) which comes next after plasma confinement problems (see for instance preliminary works of $[43,44,42,8,9])$.

On the other hand, both kinetic and fluid models, which include MHD and

\footnotetext{
${ }^{2}$ The bootstrap current comes from an magnetohydrodynamical effect and has been considered in the plasma control literature (until now) as a perturbation. However it is now hoped to be one of the major source of inductive heating in the ITER project.
} 
thermal couplings, have already been considered in the literature. Most of these models already possess a Hamiltonian structure which is considered essential by plasma physicists since the fundamental laws governing charged particle dynamics are Hamiltonian. Therefore the preservation of the Hamiltonian structure provides some confidence that the truncations that are used to derive the fluid model have not introduced unphysical phenomena. The presence of the Hamiltonian structure has the additional benefit of providing important tools for calculations such as the MHD energy principle, solvability conditions for the equilibrium equations, Casimir invariants, etc. Readers could refer to [30] for a general argumentation about Hamiltonian models for plasma physics, to [29, 26] for early work on Hamiltonian formulation of 3D kinetic tokamak model or to [46] for recent developments on the Hamiltonian formulation of a MHD fluid model. Although previous models using Hamiltonian formalisms already exist, to the best of our knowledge none of them make use of Dirac structure, nor port-variables, and they only apply to closed systems. Therefore they are not convenient for control or observation purposes.

The purpose of this paper is to propose a structured $3 \mathrm{D}^{3}$ port-Hamiltonian model for the plasma dynamics in tokamaks which is suitable for control (eventually after some symplectic reduction or discretization steps) but still captures most of the physical properties of the real plant. More precisely, we aim at representing explicitly the balance equations in the electromagnetic (EM) and "material" domains (see figure 2), their (multi-domains) closure equations and interconnection (Stokes-Dirac) structures, as well as all the possible distributed or boundary actuations (i.e. boundary energy flows). In the tokamak example, distributed and boundary actuations may occur both in the electromagnetic or "material" domains (e.g. through heat or mass injection). To build this port-Hamiltonian model, we will close the Maxwell field equations written in covariant form (see for instance [49]) with material balance equations and closure equations (including multi-domain couplings) which will be derived from the kinetic theory of gases and the Boltzmann equation (see [10]). This will require also the derivation of the irreversible entropy source term (from the GibbsDuhem relation, following the "port-based" approach in [50, Chap.3, p.154] or [14]) which is contributed by all other domains (EM, mechanic, thermal and hydraulic).

This paper is organized as follows. Section 2 recalls the port-Hamiltonian formulation for distributed parameters systems with boundary energy flows. In section 3, the electromagnetic field equations are written using this portHamiltonian formulation. In section 4 the material domain balance equations for mass, momentum and energy are derived from the Boltzmann equation (kinetic

\footnotetext{
${ }^{3}$ The chosen approach needs to identify the velocity vector field and the momentum 1 -form through the canonical Hodge star operator (see section 2.1.4 hereafter). Therefore the model is expressed in a three dimensional euclidean space. Note however that it may be reduced to a $2 \mathrm{D}$ or a 1D model by using either the axisymmetry or the quasi-static equilibrium assumptions and curvilinear coordinates $[52,6]$. Another possible approach is to consider the velocity directly as a 1-form such as in [40] for the compressible Euler equations
} 


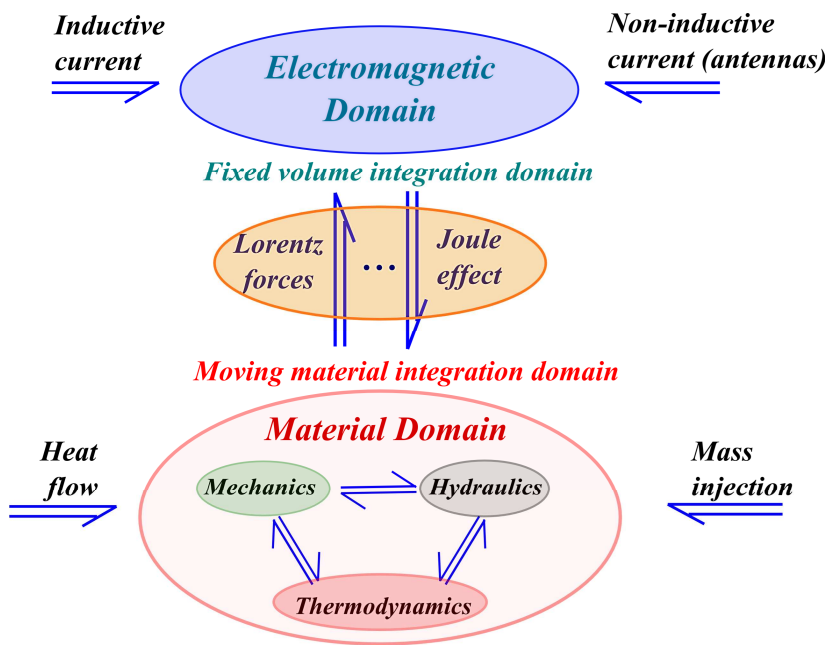

Figure 2: Model structure for the developed plasma port-Hamiltonian model. The electromagnetic balance equations are computed on a fixed volume integration domain while mass, entropy and momentum balance equations must be computed in a moving material domain. Lorentz forces and the Joule effect are the main couplings between these electromagnetic and "material" domains.

theory). The irreversible entropy production is also deduced from these three balance equations by using the Gibbs-Duhem equation. Section 5 is dedicated to closure equations which are the magneto-hydrodynamic coupling (through Lorentz forces); canonical interdomain couplings for the mechanical, hydraulical and thermal domains and a multi-domain resistivity field $(\mathcal{R}$ - field) which accounts for the entropy irreversible production. Finally, the obtained set of balance and closure equations for the plasma dynamics in a tokamak is summarized in section 6. A graphical (Bond Graph) representation of the model is proposed to underline its internal multidomain structure. The paper ends with a brief conclusion and perspectives for future works.

\section{Port-based modelling for distributed parameters systems of con- servation laws with boundary energy flows}

In this section, the port-Hamiltonian formulation for systems of conservation laws is first recalled. Port-based modelling techniques and languages have been extensively used these last decades to model, simulate and control a wide variety of lumped parameters physical systems [22, 47, 45, 23, 50, Chap.3]. More recently, an intrinsic formulation of port-Hamiltonian models for distributed parameter systems (described by partial differential state space equations) with boundary energy flow have been proposed [28, 49].

It is known that Stokes-Dirac structures allow to extend the Hamiltonian description of distributed-parameters systems to include variable boundary conditions, leading to open distributed-parameter port-Hamiltonian systems with 
boundary ports [49].

This modelling approach has been applied successfully to many hyperbolic systems as varied as transmission lines models [19], beam equations [25] or shallow water equations $[20,21]$. The same canonical Stokes-Dirac structure may be used to build port-Hamiltonian formulations of parabolic models issued from (linear) irreversible thermodynamics such as in the case of heat and mass transport phenomena in adsorption columns $[3,5]$ or ionic diffusion in fuel cell [15] or Ionic Polymer-Metal Composites [33, 32]. A more surprising parabolic plasma resistive diffusion model in the next section will show the interest of the approach in multi-physics (e.g. Thermo-MagnetoHydroDynamics) models and more involved geometries (e.g. toroidal).

\subsection{Co-variant form and exterior calculus notations}

Let us first recall some exterior calculus definitions and notations used in the sequel [16] [50, chap.4].

\subsubsection{Differential forms}

Definition 2.1. On an $n$-dimensional spatial domain $\Omega \ni \xi$, a $k$-form $(k \leq n)$, is the field $\omega_{\xi}^{k}$ of $k$-alternated forms on $T_{\xi} \Omega$, that is $k$-linear (i.e. linear with respect to each of its arguments) forms on the tangent vectors: $\omega_{\xi}^{k}:\left(T_{\xi} \Omega\right)^{k} \rightarrow \mathbb{R}$ that satisfy for any permutation $\pi$ in $\{1, . ., k\}$ and for any $k$-tuple $\left(\xi_{1}, \ldots, \xi_{k}\right) \in$ $\left(T_{\xi} \Omega\right)^{k}$ :

$$
\omega_{\xi}^{k}\left(\xi_{\pi(1)}, \ldots, \xi_{\pi(k)}\right)=\sigma(\pi) \omega_{\xi}^{k}\left(\xi_{1}, \ldots, \xi_{k}\right)
$$

where $\sigma(\pi)$ denotes the signature of the permutation $\pi$. The set of $\mathrm{k}$-forms is denoted by $\Lambda^{k}(\Omega)$.

Differential $k$-forms are endowed with a product, called exterior product, denoted by $\wedge$, which we use to compute the power and define the passivity properties, and a derivation which is called exterior derivation, denoted by d. These operators are defined as follows.

\subsubsection{Exterior product}

Consider a $k$-form $\omega^{k} \in \Lambda^{k}(\Omega)$ and a $l$-form $\omega^{l} \in \Lambda^{l}(\Omega)$, their exterior product (also called wedge product or Grassman product) is the $(k+l)$-form $\omega^{k} \wedge \omega^{l}$ :

$$
\wedge: \Lambda^{k}(\Omega) \times \Lambda^{l}(\Omega) \rightarrow \Lambda^{k+l}(\Omega)
$$

such that for any $(k+l)$-tuple of vector fields $\left(\xi_{1}, \ldots, \xi_{k+l}\right)$ :

$$
\begin{aligned}
& \left(\omega^{k} \wedge \omega^{l}\right)\left(\xi_{1}, \ldots, \xi_{k+l}\right)= \\
& \sum_{\pi \in \mathfrak{S}} \sigma(\pi) \omega^{k}\left(\xi_{\pi(1)}, \ldots, \xi_{\pi(k)}\right) \omega^{l}\left(\xi_{\pi(k+1)}, \ldots, \xi_{\pi(k+l)}\right) \in \Lambda^{k+l}(\Omega)
\end{aligned}
$$

where $\mathfrak{S}$ denotes the sets of shuffle permutation that is the permutation $\pi$ satisfying $\pi(1)<\ldots<\pi(k)$ and $\pi(k+1)<\ldots<\pi(k+l)$. 


\subsubsection{Exterior derivation}

The exterior derivation (also called co-boundary map) of a $k$-form $\omega^{k} \in$ $\Lambda^{k}(\Omega)$, denoted $\mathrm{d} \omega^{k}$, is a derivation of degree 1 which maps $\Lambda^{k}(\Omega)$ into $\Lambda^{k+1}(\Omega)$

$$
\mathrm{d}: \Lambda^{k}(\Omega) \rightarrow \Lambda^{k+1}(\Omega)
$$

and satisfies:

- linearity $\mathrm{d}(\omega+\eta)=\mathrm{d} \omega+\mathrm{d} \eta$

- anti-derivation $\mathrm{d}\left(\omega^{k} \wedge \omega^{l}\right)=(-1)^{k}\left(\mathrm{~d} \omega^{k} \wedge \omega^{l}\right)+\left(\omega^{k} \wedge \mathrm{d} \omega^{l}\right) \quad \omega^{k} \in$ $\Lambda^{k}(\Omega), \omega^{l} \in \Lambda^{l}(\Omega)$

- if $\omega^{0}$ is a smooth function, $\mathrm{d} \omega^{0}$ is the differential of the function

- $\mathrm{d} \wedge \mathrm{d}=0$

- the derivation is local: for any open set $U \subset \Omega$, if the restrictions to $U$ of two $k$-forms coincide then also the restrictions of their exterior derivatives.

Another operator associated with an inner product on the exterior differential forms is the Hodge star operator.

\subsubsection{Hodge star operator}

The Hodge star (cf. [11, V.A.,p.295]) is essential in the definition of the Hamiltonian (or energy) functions of many physical systems which admits "quadratic" energy. Assume that the vector space $\Lambda^{k}(\Omega)$ of $k$-forms on $\Omega$ is endowed with an inner product denoted by $($,$) , then the Hodge star is a linear map that$ transforms a $k$-form $\omega^{k}$ into a $(n-k)$-form, also called the dual or the pseudo $(n-k)$-form $\star \omega^{k}$ :

$$
\star: \Lambda^{k}(\Omega) \rightarrow \Lambda^{n-k}(\Omega)
$$

Thus:

$$
\left(\alpha, \omega^{k}\right)=\int_{\Omega} \alpha \wedge\left(\star \omega^{k}\right) \quad \forall \alpha \in \Omega^{k}(\Omega)
$$

In this paper, the Hodge star operator definition makes use of the canonical inner product. It also depends on the orientation defined for $\Omega$ (the sign is changed when reversing orientation). In the sequel, this Hodge star operator will be used to define constitutive closure equations which usually depend on the considered material such as Lorentz forces, Joule dissipation, viscosity, etc. We will also define the macroscopic kinetic energy of the gas as one of the constitutive equations since it must be considered in the plasma example only as an average value for kinetic energies of several species of particles with specific velocities and masses. For other examples (such as anisotropic material domain), non trivial metric must be used to define properly the corresponding Hodge star operator.

The contraction of a $k$-form with some vector field is defined hereafter. It may be seen as an anti-derivation formulae. 


\subsubsection{Contraction}

The contraction of a $k$-form $\omega^{k} \in \Lambda^{k}(\Omega)$ by some vector field $v \in T_{\xi} \Omega$, denoted by $i_{v} \omega^{k}$, is a derivation of degree -1 which maps:

$$
i_{v}: \Lambda^{k}(\Omega) \rightarrow \Lambda^{k-1}(\Omega)
$$

such that for any $(k-1)$-tuple of vector fields $\left(\xi_{1}, \ldots, \xi_{k-1}\right)$ :

$$
i_{\xi} \omega^{k}\left(\xi_{1}, \ldots, \xi_{k-1}\right)=\omega^{k}\left(\xi, \xi_{1}, \ldots, \xi_{k-1}\right)
$$

Finally we shall recall the Stokes' theorem which is fundamental for the definition of the Dirac structure underlying systems of conservation laws with energy flux at the boundary.

\subsubsection{Stokes's theorem}

Theorem 2.2. Consider a spatial domain $\Omega \in \mathbb{R}^{n}$ being an $k$-dimensional smooth manifold with smooth $(k-1)$-dimensional boundary $\partial \Omega$. Then for any $(k-1)$-form $\omega^{k-1}$ with compact support in $\mathbb{R}^{n}$, one has:

$$
\int_{\Omega} d \omega^{k-1}=\int_{\partial \Omega} \omega^{k-1}
$$

Note that from here, the spatial domain $\Omega$ is considered to be a $3 D$ domain $\Omega \subset \mathbb{R}^{3}$.

\subsection{Systems of conservation laws}

We consider here the case of systems of two conservation laws in canonical interaction.

Definition 2.3. Consider the two conserved quantities as being two 2-forms: $q \in \Lambda^{2}(\Omega)$ and $p \in \Lambda^{2}(\Omega)$. Consider also the system of conservation laws, with flux variables $\beta_{q} \in \Lambda^{1}(\Omega)$ and $\beta_{p} \in \Lambda^{1}(\Omega)$ for each conserved quantity, defined by the Hamiltonian density function $\mathcal{H}: \Lambda^{2}(\Omega) \times \Lambda^{1}(\Omega) \rightarrow \Lambda^{3}(\Omega)$ resulting in the total Hamiltonian $\mathbb{H}:=\int_{\Omega} \mathcal{H}(q, p) \in \mathbb{R}$. The system of two canonically interacting conservation laws is then defined by:

$$
\frac{\partial}{\partial t}\left(\begin{array}{c}
q \\
p
\end{array}\right)+d\left(\begin{array}{c}
\beta_{q} \\
\beta_{p}
\end{array}\right)=0 \text { and }\left(\begin{array}{c}
\beta_{q} \\
\beta_{p}
\end{array}\right)=\varepsilon\left(\begin{array}{ll}
0 & 1 \\
1 & 0
\end{array}\right)\left(\begin{array}{c}
\frac{\delta \mathcal{H}}{\delta q} \\
\frac{\delta \mathcal{H}}{\delta p}
\end{array}\right)
$$

where $\varepsilon \in\{-1,+1\}$ depends on the fluxes sign convention on the physical domain.

This system of two conservation laws may be also written as follows:

$$
\frac{\partial}{\partial t}\left(\begin{array}{c}
q \\
p
\end{array}\right)=\varepsilon\left(\begin{array}{cc}
0 & \mathrm{~d} \\
\mathrm{~d} & 0
\end{array}\right)\left(\begin{array}{c}
\frac{\delta \mathcal{H}}{\delta q} \\
\frac{\delta \mathcal{H}}{\delta p}
\end{array}\right)
$$


that is as an infinite-dimensional Hamiltonian system defined with respect to the matrix differential operator:

$$
\mathcal{J}=\varepsilon\left(\begin{array}{ll}
0 & \mathrm{~d} \\
\mathrm{~d} & 0
\end{array}\right)
$$

and generated by the Hamiltonian function $\mathbb{H}[34]$.

In order to generate a Hamiltonian systems, the matrix differential operator $\mathcal{J}$ defined in (4) should satisfy the properties of a Hamiltonian operator, that is it should be skew-symmetric and satisfy the Jacobi identities. A short calculus shows that the skew-symmetry holds only for functions with compact support in the spatial domain $\Omega$ or satisfying Dirichlet or Neumann homogeneous boundary conditions.

2.3. Stokes-Dirac structures and systems of balance equations with boundary energy flows

Interested in observation and control problems, one must consider more general (dynamic) boundary conditions where some energy is exchanged through the boundary of the spatial domain. Therefore the matrix differential operator $\mathcal{J}$ must be extended to a Dirac structure, called Stokes-Dirac structure $[49,27,24]$. Dirac structures $[12,13]$ are a geometric perspective to skewsymmetric tensors, actually corresponding to their graph, which generalize the tensors associated with Poisson brackets or pre-symplectic forms. They have been introduced in classical mechanics to represent systems with constraints. Then they have been used to include input-output port variables in finite dimensional port-Hamiltonian models [48].

Definition 2.4. Let $\mathcal{F}$ and $\mathcal{E}$ be linear spaces with a pairing $\langle e \mid f\rangle \in \mathcal{L}$ linear space, $e \in \mathcal{E}$ and $f \in \mathcal{F}$. By symmetrizing the pairing we obtain a symmetric bilinear form $\ll . \gg$ on $\mathcal{F} \times \mathcal{E}$ defined as:

$$
\ll\left(f_{1}, e_{1}\right),\left(f_{2}, e_{2}\right) \gg:=\left\langle e_{1} \mid f_{2}\right\rangle+\left\langle e_{2} \mid f_{1}\right\rangle
$$

A Dirac structure is a linear subspace $\mathcal{D} \subset \mathcal{F} \times \mathcal{E}$ such that $\mathcal{D}=\mathcal{D}^{\perp}$, with $\perp$ denoting the orthogonal complement with respect to the bilinear form $\ll . \gg$.

Making use of the Stokes theorem, this definition of Dirac structure has been used to represent canonical systems of conservation laws with boundary energy flows as described in the proposition 2.5 hereafter, defining a so-called infinite dimensional Stokes-Dirac interconnection structure [49].

Proposition 2.5. Consider the product spaces of k-forms:

$$
\begin{array}{lll}
\mathcal{F} & =\Lambda^{2}(\Omega) \times \Lambda^{2}(\Omega) \times \Lambda^{1}(\partial \Omega) \ni & \left(f_{p}, f_{q}, f_{b}\right) \\
\mathcal{E}=\Lambda^{1}(\Omega) \times \Lambda^{1}(\Omega) \times \Lambda^{1}(\partial \Omega) \ni & \left(e_{p}, e_{q}, e_{b}\right)
\end{array}
$$


Consider the linear subspace $\mathcal{D}$ of the bond space $\mathcal{B}=\mathcal{F} \times \mathcal{E}$ :

$$
\begin{aligned}
& \mathcal{D}=\left\{\left(f_{p}, f_{q}, f_{b}, e_{p}, e_{q}, e_{b}\right) \in \mathcal{F} \times \mathcal{E} \mid\right. \\
& {\left[\begin{array}{l}
f_{p} \\
f_{q}
\end{array}\right]=\varepsilon\left[\begin{array}{ll}
0 & d \\
d & 0
\end{array}\right]\left[\begin{array}{l}
e_{p} \\
e_{q}
\end{array}\right],} \\
& \left.\left[\begin{array}{l}
f_{b} \\
e_{b}
\end{array}\right]=\left[\begin{array}{cc}
\varepsilon & 0 \\
0 & -1
\end{array}\right]\left[\begin{array}{l}
e_{p \mid \partial \Omega} \\
e_{q \mid \partial \Omega}
\end{array}\right]\right\}
\end{aligned}
$$

where $\varepsilon \in\{-1,+1\}$ and $\left.\right|_{\partial \Omega}$ denotes restriction to the boundary $\partial \Omega=[0, L]$. Then $\mathcal{D}$ is a Dirac structure with respect to the non degenerated bilinear form between $\mathcal{F}$ and $\mathcal{E}$ :

$$
\left\langle\left(e_{p}, e_{q}, e_{b}\right) \mid\left(f_{p}, f_{q}, f_{b}\right)\right\rangle=\int_{\Omega}\left[e_{p} \wedge f_{p}+e_{q} \wedge f_{q}\right]+\left\langle e_{b}, f_{b}\right\rangle_{\partial \Omega}
$$

The interconnection structure $\mathcal{D}$ is called a Stokes-Dirac structure

As a consequence of proposition 2.5 one may define a Hamiltonian system with respect to this Stokes-Dirac structure as follows.

Definition 2.6. The boundary port-Hamiltonian system of two conservation laws with state space $\Lambda^{2}(\Omega) \times \Lambda^{2}(\Omega) \ni(q, p)$ and boundary port variables spaces $\Lambda^{1}(\partial \Omega) \times \Lambda^{1}(\partial \Omega) \ni\left(f_{b}, e_{b}\right)$, is the Hamiltonian system defined with respect to the Stokes-Dirac structure $\mathscr{D}$ given in proposition 2.5 and generated by the Hamiltonian functional $\mathbb{H}(q, p)$, as follows:

$$
\left(\left(\left(-\frac{\partial p}{\partial t},-\frac{\partial q}{\partial t}\right), f_{b}\right),\left(\left(\frac{\delta \mathcal{H}}{\delta p}, \frac{\delta \mathcal{H}}{\delta q}\right), e_{b}\right)\right) \in \mathcal{D}
$$

The choice of boundary conditions has obviously to be added to the definition of a boundary port-Hamiltonian system in order to define a Cauchy problem. In fact, in the linear case, a boundary port-Hamiltonian system defines a class of well-posed systems. For any solution, the isotropy condition of the Dirac structure implies the balance equation on the Hamiltonian:

$$
\frac{d \mathbb{H}}{d t}=\left\langle e_{b}, f_{b}\right\rangle_{\partial}
$$

\section{Co-variant formulation for the dynamics in the electromagnetic domain}

We detail hereafter the port-Hamiltonian co-variant formulation for electrodynamics [49] [50, chap.4]. The model is inspired from [49,31] and adapted to include additional Lorentz forces. The main used equations of electromagnetism are summarized in the Table 1 below, both in vector calculus and covariant (differential forms) notations.

The electric and magnetic field intensities are naturally identified in [49] with the 1-forms: $E, H \in \Lambda^{1}(\Omega)$; the magnetic, electric flux and free current 


\begin{tabular}{|c|c|c|}
\hline Name & Vector calculus formulation & Co-variant formulation \\
\hline \hline Gauss's law & $\nabla \cdot D=\rho_{e}$ & $d D=\rho_{e}$ \\
\hline Gauss's law for magnetism & $\nabla \cdot B=0$ & $d B=0$ \\
\hline Maxwell- Faraday & $\nabla \times E=-\frac{\partial B}{\partial t}$ & $d E=-\frac{\partial B}{\partial t}$ \\
\hline Maxwell- Ampere & $\nabla \times H=J+\frac{\partial D}{\partial t}$ & $d H=J+\frac{\partial D}{\partial t}$ \\
\hline Constitutive equations & $D=\epsilon E$ & $D=\star_{\epsilon} E$ \\
& $B=\mu H$ & $B=\star_{\mu} H$ \\
\hline
\end{tabular}

Table 1: The vector notation form and the co-variant formulation of the characteristic equations of electrodynamics. The Hodge star products $\star_{\epsilon}, \star_{\mu}$ are associated with the permittivity and permeability tensors respectively.

densities are identified with the 2-forms: $B, D, J \in \Lambda^{2}(\Omega)$; and the charge density is written as a 3-form: $\rho_{e} \in \Lambda^{3}(\Omega)$. Note that the plasma velocity $v$ is defined as a vector field, which is needed in most of the contraction notations.

The electrodynamic equations may be formulated, using the co-variant formulation [41], as a port-Hamiltonian system [49]. The Hamiltonian functional is total electromagnetic energy $\mathbb{H}(D, B)$ in the domain $\Omega$ which is the integration of the energy density $\mathcal{H}=\frac{1}{2}[E \wedge D+H \wedge B]$ :

$$
\mathbb{H}(D, B)=\int_{\Omega} \mathcal{H}=\frac{1}{2} \int_{\Omega}\left[\star_{\frac{1}{\varepsilon}} D \wedge D+\star_{\frac{1}{\mu}} B \wedge B\right]
$$

The electric and magnetic fields intensities are therefore intensive variables defined as variational derivatives of $\mathbb{H}(D, B)$, that is respectively the two 1-forms: $E, H \in \Lambda^{1}(\Omega)$ :

$$
\begin{aligned}
& \frac{\delta \mathcal{H}}{\delta D}=E(t, x) \\
& \frac{\delta \mathcal{H}}{\delta B}=H(t, x)
\end{aligned}
$$

From the power continuity and Stokes theorem, one may derive the following Stokes-Dirac structure (see [49] for the definition and details) on the space of flows $\mathcal{F}=\Lambda^{2}(\Omega) \times \Lambda^{2}(\Omega) \times \Lambda^{2}(\Omega) \times \Lambda^{1}(\partial \Omega)$ and efforts $\mathcal{E}=\Lambda^{1}(\Omega) \times \Lambda^{1}(\Omega) \times$ $\Lambda^{2}(\Omega) \times \Lambda^{1}(\partial \Omega)$ :

$$
\left.\begin{array}{c}
\mathcal{D}_{E M}= \\
\left(\begin{array}{c}
\left(f_{e l}, f_{m g}, f_{d}, f_{\partial \Omega}, e_{e l}, e_{m g}, e_{d}, e_{\partial \Omega}\right) \in \mathcal{F} \times \mathcal{E} \mid \\
f_{e l} \\
f_{m g}
\end{array}\right)=\left(\begin{array}{cc}
0 & -\mathrm{d} \\
\mathrm{d} & 0
\end{array}\right)\left(\begin{array}{c}
e_{e l} \\
e_{m g}
\end{array}\right)+\left(\begin{array}{c}
1 \\
0
\end{array}\right) f_{d}, \\
e_{d}=\left(\begin{array}{ll}
1 & 0
\end{array}\right)\left(\begin{array}{c}
e_{e l} \\
e_{m g}
\end{array}\right) \text { and }\left(\begin{array}{c}
f_{\partial \Omega} \\
e_{\partial \Omega}
\end{array}\right)=\left(\begin{array}{c}
\left.e_{e l}\right|_{\partial \Omega} \\
\left.e_{m g}\right|_{\partial \Omega}
\end{array}\right)
\end{array}\right\}
$$

where $\left.\omega^{1}\right|_{\partial \Omega}$ denotes the restriction of the 1-forms at the boundary of the domain. Then the electromagnetic field equations may be implicitly defined as a port-Hamiltonian systems:

$$
\left(-\frac{\partial D}{\partial t},-\frac{\partial B}{\partial t}, f_{d}, f_{\partial \Omega}, E, H, e_{d}, e_{\partial \Omega}\right) \in \mathcal{D}_{E M}
$$


with boundary port variables:

$$
\left\{\begin{array}{l}
f_{\partial \Omega}=\left.E\right|_{\partial \Omega} \\
e_{\partial \Omega}=\left.H\right|_{\partial \Omega}
\end{array}\right.
$$

whose wedge product forms the Poynting vector at the boundary of the spatial domain $\partial \Omega$. The distributed port variables in the domain $\Omega$ are:

$$
\left\{\begin{array}{l}
f_{d}=J \\
e_{d}=E
\end{array}\right.
$$

where $J=J_{\Omega}+J_{n i}$ is the total current density including the Ohmic and the external non-inductive current (created by the RF waves). This distributed port is terminated with the Ohm's resistive equation $\star_{\eta} J_{\Omega}$ (with $\star_{\eta}$ the Hodge star related to the plasma resistivity) and connected to the material domain via the Lorentz force equation. The Lorentz force $E_{L}$ may be written using the contraction:

$$
E_{L}=-i_{v} B
$$

and we can derive the relation:

$$
E=\star_{\eta} J_{\Omega}-i_{v} B
$$

The implicit formulation (13) reveals to be extremely useful for the systems and control theory [50]. Among others, it satisfies the following energy balance equation:

$$
\frac{d \mathbb{H}}{d t}=-\int_{\partial \Omega} e_{\partial \Omega} \wedge f_{\partial \Omega}-\int_{\Omega} e_{d} \wedge f_{d}
$$

In other words, with the appropriate choice of input and output variables, the system passivity is easily proved and the energy evolution is governed by the external energy supplied through the boundary (and by the Ohmic dissipation rate).

A commonly used control model for plasma current density profiles in tokamaks is the so-called $1 \mathrm{D}$ resistive diffusion equation [6]. This $1 \mathrm{D}$ model may be derived from the previous 3D model by using the usual axisymmetry and quasi-static assumptions (the Maxwell-Ampere and Maxwell-Faraday equations may be written in toric magnetic coordinates in the form of a system where all variables depends only on the magnetic surface radius $\rho$ ). In [51], a symplectic spectral discretization is proposed to validate this $1 \mathrm{D}$ control model.

The influences of the dynamics in material domain on certain parameters in the electromagnetic domain, like the resistivity $\eta$ and the bootstrap noninductive bootstrap current $J_{b t}$, is ignored in this resistive diffusion equation and in the many control studies which make use of it. In the sequel, we determine first the material balance equations and then the coupling between the electromagnetic and material domains which allows to include the dependance of the resistivity (and of the diffusivity) with the temperature and induction field profiles. The bootstrap current still remains an unmodelled source term and its explicit formulation in the model (as an inter-domain coupling) is among the prospects of this work. 


\section{Balance and closure equations in the material domain}

In this section, the material balance equations for mass, momentum, energy and entropy are derived from the Boltzmann equation (in kinetic theory). These balance equations are computed for a moving material domain. The connection between the classical macroscopic transport equation and the port-based formulation is made by using the material derivative in co-variant form and the Gibbs-Duhem relation for the internal energy (cf. [50, Chap. 3, p. 154]). The result is a port-based fluid-like model expressed in terms of pairs of power conjugated variables.

\subsection{Kinetic theory and macroscopic transport equations}

Using a kinetic description for the plasma dynamics, the material balance equations (mass, momentum, and energy balances) may be obtained from the integrals of the Boltzmann equation. These computations are usually performed in chosen coordinate systems for a 3D Euclidean space (see e.g. [10, p. 205]). Therefore, in this subsection, we recall the classical results obtained using the Nabla operator to define the grad, curl and div operators respectively as $(\nabla, \nabla \times, \nabla$.$) , before stating the covariant formulation in subsection 4.2.$

\subsubsection{Boltzmann equation}

The Boltzmann equation is a state equation which describes the time evolution of the distribution functions $f_{a}(t, r, v)$ for particles with position $r$ and velocity $v$ in the coordinate system $\beta$ :

$$
\frac{\partial f_{a}}{\partial t}+\frac{\partial}{\partial r_{\beta}}\left(v_{\beta} f_{a}\right)+\frac{\partial}{\partial v_{\beta}}\left(\frac{F_{a \beta}}{m_{a}} f_{a}\right)=C_{a}
$$

Here the subscript $a$ stands for the different considered species, such as electrons $a=e$, ions $a=i$, or neutral particles $a=n$ in our case. The external force:

$$
F_{a}=e_{a} E+\frac{e_{a}}{c}[v B]
$$

is caused by electric and magnetic fields, respectively $E$ and $B$. The collision term is usually of the form $C_{a}=\sum_{b} C_{a b}\left(f_{a}, f_{b}\right)$ where $C_{a b}$ denotes the rate of change for the distribution function of particles $a$ due to elastic and/or inelastic collisions with $b$. This collision term is neglected in this work. It is a quite usual assumption since the plasma gas in tokamaks is low density and usually considered as a perfect gas (cf. [10, p.206] or [6, Chap.6]). Averaging momenta of the distribution function over all possible velocities, the average or macroscopic 
classical (fluid-like) quantities may be derived:

$$
\begin{aligned}
n_{a}(t, r) & =\int_{\mathbb{R}^{3}} f_{a}(t, r, v) d v \\
\bar{v}_{a}(t, r) & =\frac{1}{n_{a}} \int_{\mathbb{R}^{3}} v f(t, r, v) d v=\langle v\rangle_{a} \\
T_{a}(t, r) & =\frac{1}{n_{a}} \int_{\mathbb{R}^{3}} \frac{m_{a}}{3}\left(v-\bar{v}_{a}\right)^{2} f(t, r, v) d v \\
& =\frac{m_{a}}{3}\left\langle\left(v-\bar{v}_{a}\right)^{2}\right\rangle
\end{aligned}
$$

which are respectively the $a$-particles density, average velocity and temperature. In (18) the integrals' domain has been chosen to be $\mathbb{R}^{3}$ because no restrictions are considered on the admissible velocities. The bracket $\langle$.$\rangle denotes the aver-$ age value of a variable, it should be distinguished with the paring $\langle.,$.$\rangle using$ in covariant form. The third equation is obtained by considering the thermal equilibrium (when $\left.\bar{v}_{a}=0\right) m\left\langle v^{2}\right\rangle / 2=3 T / 2$. In this work, there is no fusion reaction considered. Hence balance equations for one species in the plasma may be usually derived from those for the other species (neglecting the eventual interaction terms). Macroscopic variables related to only one representative species will be considered in this work. Therefore, there will be no more use of the species subscripts $a$ for electrons, ions, and neutral particles. In other words, $T, P, v, \ldots$ will be representatives of average temperature, pressure, velocity, ... for all species (which usually correspond to electronic values in the plasma control community).

\subsubsection{Transport equations (balance equations)}

Three macroscopic balance equations may be derived by multiplying the Boltzmann equation (16) with $1, m v$, and $\frac{m v^{2}}{2}$ respectively, and integrating the results over the velocity domain (the detailed derivation, inspired from cf. [10, p.208] may be found in the appendix). Defining the usual material derivative by

$$
\frac{d}{d t}=\frac{\partial}{\partial t}+\bar{v} \cdot \nabla
$$

these calculations lead to:

- the equation of continuity (particle transport):

$$
\frac{d n}{d t}=-n \nabla \cdot \bar{v}
$$

which is obtained assuming the density conservation (no external source term) and the assumption that the third term in (16) vanishes rapidly as $v \rightarrow \infty$. 
- the equation of motion (momentum transport):

$$
n \frac{d \bar{v}}{d t}=-\nabla P-\nabla \times \tau-e n\left(E+\frac{1}{c}[\bar{v} B]\right)
$$

where $P$ denotes the scalar pressure, $\tau$ the stress tensor, and electron charge $e=1.610^{-19} \mathrm{C}$.

- the (total) energy transport equation:

$$
\frac{d \epsilon}{d t}=-\nabla \cdot[n T \bar{v}+\tau \bar{v}+\mathbf{q}]+e n(E \bar{v})
$$

where $\mathbf{q}$ is defined as the "heat" flux density, $T$ is the average temperature and the total energy is defined as:

$$
\epsilon=\frac{m n}{2} \bar{v}^{2}+\frac{3}{2} n T
$$

The internal energy (or heat balance) equation may be obtained when the total energy (23) is replaced in equation (22), using the continuity and momentum equations:

$$
\frac{3}{2} n \frac{d T}{d t}=-P \nabla \cdot \bar{v}-\nabla \cdot \mathbf{q}-\tau(\nabla \times \bar{v})
$$

Defining the entropy in the perfect gas case as $\mathbf{s}=\ln \left(T^{3 / 2} / n\right)=\ln \left(P^{3 / 2} / n^{5 / 2}\right)$, the heat balance equation may be written:

$$
\operatorname{Tn} \frac{d \mathbf{s}}{d t}=-\nabla \cdot \mathbf{q}-\tau(\nabla \times \bar{v})
$$

The balance equations (20), (21), (24) and (25) ) will now be written in co-variant form for a representation in the port-Hamiltonian formulation.

\subsection{Co-variant formulation of the transport equations}

The properties and dynamics of the plasma considered as a fluid will be described in this section. The mass, momentum, energy and entropy balance equations will be derived in the co-variant form. We shall follow closely the general frame presented in [50, Chap.3, p.154] but express the results in terms of differential forms.

The material properties are defined by the total material energy $\mathbb{H}_{M}$ which is the integral over the material domain $\mathcal{M}$ of total energy density $\epsilon$. It is the sum of the kinetic and internal energy of the system which depends on the specific quantity (per unit mass) of the momentum density $\mathbf{p} \in \Lambda^{2}(\mathcal{M})^{4}$, the entropy $\mathbf{s} \in \Lambda^{3}(\mathcal{M})$ and the volume $\mathbf{v} \in \Lambda^{3}(\mathcal{M})$.

This subsection gives a definition of the material derivative in terms of differential form which will be used in the following to write the balance equations on a moving material domain.

\footnotetext{
${ }^{4}$ The conjugated variable of the momentum density $\star \mathbf{p} \in \Lambda^{1}(\mathcal{M})$ is a 1-form equivalent to the average plasma velocity $v$ in [50, section 3.4]
} 


\subsubsection{Time derivative "following the motion"}

The material properties must be described for a fixed amount of mass which (due to some velocity field) occupies a moving material domain. We denote $\phi_{t}$ the flow associated with the velocity field $v$ (i.e. $v=d / d t\left(\phi_{t} z\right)$ ). Any moving material domain with a fixed mass may be defined by $V(t)=\phi_{t}(\Omega)$ where $\Omega$ denotes the same material domain at time $t=0$. The total variation of some conserved material quantity $\alpha^{k}$ (where $\alpha^{k}$ is a $k$-form, defined on a $n$-dimensional spatial domain ) becomes [16, Chap.1, sec.4]:

$$
\frac{d}{d t} \int_{V(t)} \alpha=\int_{V(t)} \frac{\partial \alpha}{\partial t}+\mathfrak{L}_{v} \alpha
$$

where $\mathfrak{L}_{v} \alpha$ denotes the Lie derivative of the $k$-form $\alpha$. Using Cartan's formula:

$$
\mathfrak{L}_{v}=i_{v} \circ \mathrm{d}+\mathrm{d} \circ i_{v}
$$

one may define the material derivative $\frac{D \alpha}{D t}$ in term of the variation of some conserved quantity $\alpha$ such that:

$$
\int_{V(t)} \frac{D \alpha}{D t}=\frac{d}{d t} \int_{V(t)} \alpha
$$

Therefore:

$$
\frac{D \alpha}{D t}:=\frac{\partial \alpha}{\partial t}+i_{v} \mathrm{~d} \alpha+\mathrm{d}\left(i_{v} \alpha\right)
$$

For a volume form $\alpha^{n}$ in an $n$-dimensional spatial domain $\left(\mathrm{d} \alpha^{n}=0\right)$ and (29) becomes:

$$
\frac{D}{D t} \alpha=\frac{\partial \alpha}{\partial t}+\mathrm{d} i_{v} \alpha
$$

which is equivalent to the usual $\partial_{t}+v \cdot \nabla$ vectorial form.

\subsubsection{Balance and closure equations}

A balance equation for the material quantity $\alpha$ in a fixed frame generally takes the form:

$$
\frac{\partial \alpha}{\partial t}=-\mathrm{d} f_{\alpha}+\sigma_{\alpha}
$$

where $f_{\alpha}$ defines the flux of $\alpha$, and $\sigma_{\alpha}$ represents the external $\alpha$ source (by unit of mass, since $\alpha$ is a density). Therefore, when considering a moving material domain, the balance equation becomes (using (30) and (31)):

$$
\frac{D}{D t} \alpha=-\mathrm{d} f_{\alpha}^{R}+\sigma_{\alpha}
$$

where $f_{\alpha}^{R}=f_{\alpha}-i_{v} \alpha$ is now the relative flux of $\alpha$ and $\frac{D}{D t}$ the material derivative. We can now express in co-variant form all the balance equations obtained from the kinetic theory in a moving material domain. 
Mass balance (continuity equation).

$$
\frac{\partial \rho}{\partial t}=-\mathrm{d} f_{\rho}+\sigma_{\rho}
$$

or using (20) and (32):

$$
\frac{D}{D t} \rho=-\mathrm{d} f_{\rho}^{R}+\sigma_{\rho}
$$

with the mass density $\rho=m n$, the relative particle flux $\mathrm{d} f_{\rho}^{R}=\mathrm{d}\left(f_{\rho}-i_{v} \rho\right)$, and the material source term $\sigma_{\rho}$ per unit volume.

Remark 4.1. The same continuity equation may be written as a massic volume balance equation. Let $\mathbf{v}:=1 / \rho$ denotes the specific massic volume and $\sigma_{\mathbf{v}}$ as the massic source term, then from equation (34) we obtain

$$
\frac{D}{D t} \mathbf{v}=d\left(i_{v} \mathbf{v}\right)+\sigma_{\mathbf{v}}
$$

with the relative volume flux $d f_{\mathbf{v}}^{R} \equiv d\left(-i_{v} \mathbf{v}\right)$.

Momentum balance.

$$
\frac{1}{\mathbf{v}} \frac{D \mathbf{p}}{D t}=-\mathrm{d} f_{\mathbf{p}}^{R}+\sigma_{\mathbf{p}}
$$

where $\mathbf{p}$ is the momentum per unit mass and $\sigma_{\mathbf{p}}$ is the source term caused by external body force per unit mass (the electromagnetic pressure in this work). Note that one could also use a more usual identification of both the velocity $v$ and the current $J$ as vector fields, leading to an alternative expression for the electromagnetic pressure:

$$
\sigma_{\mathbf{p}}=-i_{(\star J)} B
$$

Energy balance.

$$
\frac{D \epsilon}{D t}=-\mathrm{d} f_{\epsilon}^{R}+\sigma_{\epsilon}
$$

where the source term $\sigma_{\epsilon}$ represents the inductive (ohmic) heat source term which is $\mathbf{v}(E \wedge J)$.

\subsection{Closure equations and entropy production}

In this subsection, we consider the closure equations for the momentum and energy balance equations. Then, since the entropy is not a conserved quantity, the irreversible entropy production term must be computed.

\subsubsection{Momentum}

We first consider the momentum closure equation. According to (21), the tensor $f_{\mathbf{p}}^{R}$, representing the momentum flux per unit of surface area, is the sum of a pressure $P$ and viscous force terms $\tau$ :

$$
f_{\mathbf{p}}^{R}=(P \mathbf{I}+\tau)
$$

Considering that $P$ is a scalar (in an isotropic domain), while $\tau \in \Lambda^{1}$ is the non-diagonal part of stress tensor, or the so-called shear stress tensor, we have:

$$
\mathrm{d} f_{\mathbf{p}}^{R}=\mathrm{d} P+\star \mathrm{d} \tau
$$




\subsubsection{Internal energy}

The total energy density (per unit mass) is:

$$
\epsilon=\frac{\star \mathbf{p} \wedge \mathbf{p}}{2}+\mathbf{u}
$$

with $\mathbf{u}$ is the internal energy. Its time variation is defined according to:

$$
\begin{aligned}
\frac{D \epsilon}{D t} & =\star \mathbf{p} \wedge \frac{D \mathbf{p}}{D t}+\frac{D \mathbf{u}}{D t} \\
& =i_{v} \mathbf{v} \wedge \frac{1}{\mathbf{v}} \frac{D \mathbf{p}}{D t}+\frac{D \mathbf{u}}{D t}
\end{aligned}
$$

where the contraction $i_{v} \mathbf{v}$ represents to the plasma velocity $\mathbf{v}(\star \mathbf{p})$ of a plasma volume $\mathbf{v}$. The material derivative of internal energy $\mathbf{u}$ is written following the general formulation (32):

$$
\frac{D}{D t} \mathbf{u}=-\mathrm{d} f_{\mathbf{u}}^{R}+\sigma_{\mathbf{u}}
$$

Using the momentum balance equation (36) and the internal energy balance equation (43), the total energy balance(42) becomes:

$$
\begin{aligned}
\frac{D \epsilon}{D t} & =i_{v} \mathbf{v} \wedge\left(-\mathrm{d} f_{\mathbf{p}}^{R}+\sigma_{\mathbf{p}}\right)+\left(-\mathrm{d} f_{\mathbf{u}}^{R}+\sigma_{\mathbf{u}}\right) \\
& =-\mathrm{d}\left(i_{v} \mathbf{v} \wedge f_{\mathbf{p}}^{R}+f_{\mathbf{u}}^{R}\right)+f_{\mathbf{p}}^{R} \wedge \mathrm{d}\left(i_{v} \mathbf{v}\right)+i_{v} \mathbf{v} \wedge \sigma_{\mathbf{p}}+\sigma_{\mathbf{u}}
\end{aligned}
$$

with

$$
\left\{\begin{aligned}
f_{\mathbf{u}}^{R} & =f_{\epsilon}^{R}-i_{v} \mathbf{v} \wedge f_{\mathbf{p}}^{R} \\
\sigma_{\mathbf{u}} & =\sigma_{\epsilon}-i_{v} \mathbf{v} \wedge \sigma_{\mathbf{p}}-f_{\mathbf{p}}^{R} \wedge \mathrm{d}\left(i_{v} \mathbf{v}\right) \\
& =\mathbf{v}\left({ }_{\star \eta} J \wedge J_{t o t}\right)-\left(P \wedge \mathrm{d}\left(i_{v} \mathbf{v}\right)+\tau \wedge \mathrm{d}\left(i_{v} \mathbf{v}\right)\right)
\end{aligned}\right.
$$

\subsubsection{Irreversible entropy production}

The internal energy is also determined by the Gibbs-Duhem equation:

$$
\mathrm{d} \mathbf{u}=T \wedge \mathrm{d} \mathbf{s}-P \wedge \mathrm{d} \mathbf{v}
$$

which will allow us to compute the irreversible entropy production source term $\sigma_{s}$ (without nuclear fusion reaction in the studied case). The entropy production rate is then:

$$
\frac{D \mathbf{s}}{D t}=\frac{1}{T}\left(\frac{D \mathbf{u}}{D t}+P \wedge \frac{D \mathbf{v}}{D t}\right)
$$

Applying the material derivative formula for $\mathbf{s}, \mathbf{u}$ and $\mathbf{v}$ in (47), we get: 


$$
\begin{aligned}
\frac{D}{D t} \mathbf{s} \quad= & \frac{1}{T}\left(\frac{D u}{D t}+P \wedge \frac{D \mathbf{v}}{D t}\right) \\
\Leftrightarrow-\mathrm{d} f_{\mathbf{s}}^{R}+\sigma_{\mathbf{s}} \quad= & \frac{1}{T}\left(-\mathrm{d} f_{u}^{R}+\sigma_{\mathbf{u}}+P \wedge \mathrm{d}\left(i_{v} \mathbf{v}\right)\right) \\
\Leftrightarrow-\mathrm{d}\left(\frac{f_{q}}{T}\right)+\sigma_{\mathbf{s}}= & \frac{1}{T}\left(-\mathrm{d} f_{q}-\left(P \wedge \mathrm{d}\left(i_{v} \mathbf{v}\right)+\tau \wedge \mathrm{d}\left(i_{v} \mathbf{v}\right)\right)\right)+ \\
& +\frac{1}{T}\left(\mathbf{v}\left({ }_{\star} J \wedge J_{t o t}\right)+P \wedge \mathrm{d}\left(i_{v} \mathbf{v}\right)\right)
\end{aligned}
$$

where the heat flux $f_{q}$ is related to the entropy and internal energy fluxes:

$$
\left\{\begin{array}{l}
f_{\mathbf{u}}^{R}=f_{q} \\
f_{\mathbf{s}}^{R}=\frac{1}{T} f_{q}
\end{array}\right.
$$

Thus the irreversible entropy source term $\sigma_{\mathbf{s}}$ is obtained with the help of the internal energy source term in equation (45):

$$
\begin{aligned}
& \sigma_{\mathbf{s}}=\mathrm{d}\left(\frac{f_{q}}{T}\right)+\frac{1}{T}\left(-\mathrm{d} f_{q}-\tau \wedge \mathrm{d}\left(i_{v} \mathbf{v}\right)+g \wedge f_{\rho}^{R}\right)
\end{aligned}
$$

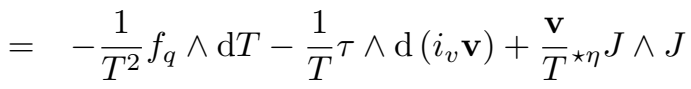

This irreversible entropy source contains the heat conduction term $-\frac{1}{T^{2}} f_{q} \wedge$ $\mathrm{d} T$, the viscous dissipation term $-\frac{1}{T} \tau \wedge \mathrm{d}\left(i_{v} \mathbf{v}\right)$ and the Joule (ohmic) term $\frac{\mathbf{v}}{T} \star \eta J \wedge J$. The three of them define the constitutive relations for the $\mathcal{R}$ - field (see next section).

\section{Multidomain couplings and resistivity field ( $\mathcal{R}$ - field)}

In this section, all power conservative multi-domain couplings are detailed (and represented using Dirac interconnection structures [49]) as well as a dissipative $\mathcal{R}$ - field multi-domain coupling element.

\subsection{Magneto-hydrodynamic coupling (MHD coupling)}

The MHD coupling is the central multi-domain couplings in the tokamak system. It may be represented using a magnetomotive Dirac structure combined with an Eulerian-Lagrangian coordinate transformation used to relate the volumetric and massic domains. 


\subsubsection{Magnetomotive coupling}

Let us now prove that the two relations (14) and (37) may be associated with a Dirac structure. We will make use of the flow and effort variables identifications $\left(f_{J}, e_{J}\right)=\left(J, E_{L}\right)$ and $\left(f_{v}, e_{v}\right)=\left(\sigma_{\mathbf{p}}, \mathbf{p}\right)$. Let $\mathcal{F}_{1}=\Lambda^{2}(\Omega) \times \Lambda^{1}(\Omega) \ni$ $\left(f_{J}, f_{v}\right)$ and $\mathcal{E}_{1}=\Lambda^{1}(\Omega) \times \Lambda^{2}(\Omega) \ni\left(e_{J}, e_{v}\right)$ be respectively the spaces of conjugated flow and effort variables. For a given magnetic induction field $B$, we can note that equations (37) and (15) define two anti-adjoint maps:

$$
\begin{aligned}
\Lambda^{2}(\Omega) & \rightarrow \Lambda^{1}(\Omega) \\
f_{J} & \mapsto f_{v}=-\star\left(\star f_{J} \wedge \star B\right)
\end{aligned}
$$

and

$$
\begin{aligned}
\Lambda^{1}(\Omega) & \rightarrow \Lambda^{2}(\Omega) \\
e_{v} & \mapsto \star\left(\star e_{v} \wedge \star B\right)
\end{aligned}
$$

Proposition 5.1. For any given $B \in \Lambda^{2}(\Omega)$, the linear subspace $\mathcal{D}_{1}(B)$

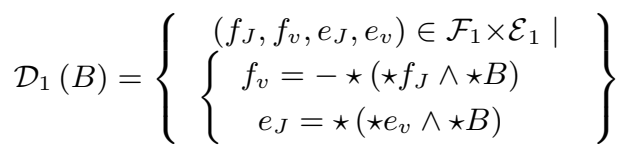

is a Dirac structure in the bond space in $\mathcal{F}_{1} \times \mathcal{E}_{1}$.

Let us first precise that the Dirac structure is defined with respect to the symmetrized pairing of the power pairing on $\mathcal{F}_{1} \times \mathcal{E}_{1}$ defined by:

$$
\left\langle\left(\begin{array}{c}
e_{J} \\
e_{v}
\end{array}\right),\left(\begin{array}{c}
f_{J} \\
f_{v}
\end{array}\right)\right\rangle=\int_{\Omega} e_{v} \wedge f_{v}-e_{J} \wedge f_{J}
$$

First note that $\mathcal{D}_{1}(B) \subset \mathcal{D}_{1}(B)^{\perp}$ (see subsection 2.3) is equivalent to:

$$
\left\langle\left(\begin{array}{c}
e \\
e_{v}
\end{array}\right),\left(\begin{array}{c}
f_{J} \\
f_{v}
\end{array}\right)\right\rangle=0, \quad \forall\left(f_{J}, f_{v}, e_{J}, e_{v}\right) \in \mathcal{F}_{1} \times \mathcal{E}_{1}
$$

which easily follows from (37). Now let us prove that $\mathcal{D}_{1}(B)^{\perp} \subset \mathcal{D}_{1}(B)$. For this, assume that $\left(f_{J}, f_{v}, e_{J}, e_{v}\right) \in \mathcal{F}_{1} \times \mathcal{E}_{1}$ satisfies $\forall\left(f_{J}^{\prime}, f_{v}^{\prime}, e_{J}^{\prime}, e_{v}^{\prime}\right) \in \mathcal{D}_{1}(B)$ :

$$
\left\langle\left(\begin{array}{c}
e_{J}^{\prime} \\
e_{v}^{\prime}
\end{array}\right),\left(\begin{array}{c}
f_{J} \\
f_{v}
\end{array}\right)\right\rangle+\left\langle\left(\begin{array}{c}
e_{J} \\
e_{v}
\end{array}\right),\left(\begin{array}{c}
f_{J}^{\prime} \\
f_{v}^{\prime}
\end{array}\right)\right\rangle=0
$$

Then, equivalently $\forall\left(f_{J}^{\prime}, e_{v}^{\prime}\right) \in \Lambda^{2}(\Omega) \times \Lambda^{1}(\Omega)$ :

$$
\left\langle\left(\begin{array}{c}
\star\left(\star e_{v}^{\prime} \wedge \star B\right) \\
e_{v}^{\prime}
\end{array}\right),\left(\begin{array}{c}
f_{J} \\
f_{v}
\end{array}\right)\right\rangle+\left\langle\left(\begin{array}{c}
e_{J} \\
e_{v}
\end{array}\right),\left(\begin{array}{c}
f_{J}^{\prime} \\
-\star\left(\star f_{J}^{\prime} \wedge \star B\right)
\end{array}\right)\right\rangle=0
$$

That is:

$$
\int_{\Omega}\left(e_{v}^{\prime} \wedge f_{v}-\star\left(\star e_{v}^{\prime} \wedge \star B\right) \wedge f_{J}\right)-\left(e_{J} \wedge f_{J}^{\prime}-e_{v} \wedge \star\left(\star f_{J}^{\prime} \wedge \star B\right)\right)=0
$$


$\forall\left(f_{J}^{\prime}, e_{v}^{\prime}\right) \in \Lambda^{2}(\Omega) \times \Lambda^{1}(\Omega)$. Using the fact that $\int_{\Omega} \star \alpha \wedge \beta=\int_{\Omega} \alpha \wedge \star \beta$ that $\star \star \alpha=\alpha$ one gets:

$$
\begin{aligned}
\int_{\Omega} \star\left(\star e_{v}^{\prime} \wedge \star B\right) \wedge f_{J} & =\int_{\Omega} \star e_{v}^{\prime} \wedge \star B \wedge \star f_{J} \\
& =-\int_{\Omega} \star e_{v}^{\prime} \wedge \star f_{J} \wedge \star B \\
& =-\int_{\Omega} e_{v}^{\prime} \wedge \star\left(\star f_{J} \wedge \star B\right)
\end{aligned}
$$

Choosing $f_{J}^{\prime}=0$, the preceding equation becomes:

$$
\int_{\Omega} e_{v}^{\prime} \wedge\left(f_{v}+\star\left(\star f_{J} \wedge \star B\right)\right)=0, \quad \forall e_{v}^{\prime} \in \Lambda^{1}(\Omega)
$$

hence $f_{v}=-\star\left(\star f_{J} \wedge \star B\right)$. In a similar way, when choosing $e_{v}^{\prime}=0$, (52) becomes:

$$
\int_{\Omega}\left(e_{J} \wedge f_{J}^{\prime}+e_{v} \wedge \star\left(\star f_{J}^{\prime} \wedge \star B\right)\right)=0, \quad \forall f_{J}^{\prime} \in \Lambda^{2}(\Omega)
$$

or with similar calculus:

$$
\begin{aligned}
& \int_{\Omega} e_{v} \wedge \star\left(\star f_{J}^{\prime} \wedge \star B\right)=-\int_{\Omega} e_{v} \wedge \star\left(\star B \wedge \star f_{J}^{\prime}\right) \\
& =-\int_{\Omega} \star e_{v} \wedge \star B \wedge \star f_{J}^{\prime} \\
& =-\int_{\Omega}\left(\star e_{v} \wedge \star B\right) \wedge \star f_{J}^{\prime} \\
& =-\int_{\Omega} \star\left(\star e_{v} \wedge \star B\right) \wedge f_{J}^{\prime} \\
& \int_{\Omega}\left(e_{J}-\star\left(\star e_{v} \wedge \star B\right) \wedge f_{J}^{\prime}\right) \wedge f_{J}^{\prime}=0, \quad \forall f_{J}^{\prime} \in \Lambda^{2}(\Omega)
\end{aligned}
$$

yielding $e_{J}=\star\left(\star e_{v} \wedge \star B\right)$. Hence $\left(f_{J}, f_{v}, e_{J}, e_{v}\right) \in \mathcal{D}_{1}(B)$ which completes the proof.

\subsubsection{Eulerian-Lagrangian transformation}

We shall define hereafter a Dirac structure associated with the change from Eulerian to Lagrangian coordinates (that is from integration on a fixed domain $\Omega$ to a moving domain $M=\phi_{t}(\Omega)$ ). Therefore let us write the power product between the effort and the flow variables in the two coordinates and look for the following equality:

$$
\int_{M=\phi_{t}(\Omega)} e_{2} \wedge f_{2}=\int_{\Omega} \phi^{*}\left(e_{2} \wedge f_{2}\right)=\int_{\Omega} \phi^{*}\left(e_{2}\right) \wedge f_{1}
$$

with $\phi^{*}\left(e_{2}\right)=1 / \star \mathbf{v} e_{2} \circ \phi^{-1}$, where $\mathbf{v}$ is the specific massic volume of the plasma. This defines the following vector subspace :

$$
\mathcal{D}(\mathbf{v})=\left\{\begin{array}{c}
\left(f_{1}, f_{2}, e_{1}, e_{2}\right) \in\left(\Lambda^{1}(\Omega) \times \Lambda^{1}(M)\right) \times\left(\Lambda^{2}(\Omega) \times \Lambda^{2}(M)\right) \\
f_{2}=f_{1} \circ \phi^{-1} ; e_{1}=\phi^{*}\left(e_{2}\right) \circ \phi ; \\
f_{1} \in \Lambda^{1}(\Omega), e_{2} \in \Lambda^{2}(\phi(\Omega))
\end{array}\right\}
$$

The subspace $\mathcal{D}(\mathbf{v})$ (as well as the subspace resulting from the interconnection of $\mathcal{D}_{1}$ and $\left.\mathcal{D}(\mathbf{v})\right)$ may be proved to be Dirac structure using the power 
product in the material domain suggested in (53) and following arguments similar to those in [49]. In the tokamak model the pairs of flow and effort variables connected to the Dirac interconnection structures $\mathcal{D}_{1}$ and $\mathcal{D}(\mathbf{v})$ are respectively $\left(f_{1}, e_{1} ; f_{2}, e_{2}\right)_{1}=\left(-i_{\star J} B, \mathbf{p} ;-i_{\star J} B, i_{v} \mathbf{v}\right)$ and $\left(f_{1}, e_{1} ; f_{2}, e_{2}\right)_{2}=$ $\left(J,{ }_{\star \eta} J ; J,{ }_{\star \eta} J \mathbf{v}\right)$.

\subsection{Interconnection structures in the material domains}

The material domains are the mechanical, hydraulical and thermal domains. The pairs of energy variables in these subdomains are interconnected together through Stokes-Dirac structures of the form:

$$
\mathcal{D}_{i}=\left\{\begin{array}{c}
\left(f_{p}, f_{q}, f_{i \partial M}, e_{p}, e_{q}, e_{i \partial M}\right) \in \mathcal{F}_{\mathrm{i}} \times \mathcal{E}_{\mathrm{i}} \mid \\
\left(\begin{array}{c}
f_{p} \\
f_{q}
\end{array}\right)=\left(\begin{array}{cc}
0 & \mathrm{~d} \\
\mathrm{~d} & 0
\end{array}\right)\left(\begin{array}{c}
e_{p} \\
e_{q}
\end{array}\right), \\
\left(\begin{array}{c}
f_{i \partial M} \\
e_{i \partial M}
\end{array}\right)=\left.\left(\begin{array}{c}
1 \\
1
\end{array}\right)\left(\begin{array}{c}
e_{p} \\
e_{q}
\end{array}\right)\right|_{\partial M}
\end{array}\right\} i=\{2,3,4\}
$$

- The Stokes-Dirac structure $\mathcal{D}_{2}$ for the hydraulical domain is:

$\left(-\mathrm{d} P, \mathrm{~d}\left(i_{v} \mathbf{v}\right), f_{2 \partial M}, i_{v} \mathbf{v},-P, e_{2 \partial M}\right)=\left(f_{p}, f_{q}, f_{2 \partial M}, e_{p}, e_{q}, e_{2 \partial M}\right) \in \mathcal{F}_{2} \times \mathcal{E}_{2}$

where $\left\{\begin{array}{l}\mathcal{F}_{2}=\Lambda^{1}(M) \times \Lambda^{3}(M) \times \Lambda^{0}(M) \\ \mathcal{E}_{2}=\Lambda^{2}(M) \times \Lambda^{0}(M) \times \Lambda^{2}(M)\end{array}\right.$

- The Stokes-Dirac structure $\mathcal{D}_{3}$ for the mechanical domain may be written:

$$
\left(\mathrm{d} \tau, \mathrm{d}\left(i_{v} \mathbf{v}\right), f_{3 \partial M}, i_{v} \mathbf{v}, \tau, e_{3 \partial M}\right)=\left(f_{p}, f_{q}, f_{3 \partial M}, e_{p}, e_{q}, e_{3 \partial M}\right) \in \mathcal{F}_{3} \times \mathcal{E}_{3}
$$

where $\left\{\begin{array}{l}\mathcal{F}_{3}=\Lambda^{2}(M) \times \Lambda^{2}(M) \times \Lambda^{1}(M) \\ \mathcal{E}_{3}=\Lambda^{1}(M) \times \Lambda^{1}(M) \times \Lambda^{1}(M)\end{array}\right.$

- The Fourrier heat transport in the thermal domain may also be represented by using a Stokes-Dirac structure $\mathcal{D}_{4}$ of the form (see for instance [4], here the exterior derivative of $T$ is a spatial gradient):

$$
\left(\mathrm{d}\left(\frac{f_{q}}{T}\right), \mathrm{d} T, f_{4 \partial M}, T,\left(\frac{f_{q}}{T}\right), e_{4 \partial M}\right)=\left(f_{p}, f_{q}, f_{3 \partial M}, e_{p}, e_{q}, e_{3 \partial M}\right) \in \mathcal{F}_{4} \times \mathcal{E}_{4}
$$

where $\left\{\begin{array}{l}\mathcal{F}_{4}=\Lambda^{3}(M) \times \Lambda^{1}(M) \times \Lambda^{2}(M) \\ \mathcal{E}_{4}=\Lambda^{0}(M) \times \Lambda^{2}(M) \times \Lambda^{0}(M)\end{array}\right.$

\subsection{Multi-domains fields elements}

In this section we formally introduce a dissipative $\mathcal{R}$ - field accounting for the irreversible entropy production (50) and a conservative $\mathcal{C}$ - field which represents the first principle in the material domain with the energy density given in (41). We follow a classical approach from irreversible (near equilibrium) thermodynamics [35] and apply it to model transport phenomena in toroidal plasmas. Therefore we will assume the existence of a (semi) linear relation between the generalized flows and thermodynamical forces. 


\subsubsection{Resistive field $(\mathcal{R}$ - field)}

According to (50), the irreversible entropy production $\sigma_{s}$ creation is contributed by different resistivity sources: the heat conduction, the ohmic diffusion and the viscosity, with associated dissipated power:

$$
T \sigma_{s}=-\frac{1}{T} f_{q} \wedge \mathrm{d} \mathrm{T}-\tau \wedge \mathrm{d}\left(i_{v} \mathbf{v}\right)+\mathbf{v}\left(\star_{\eta} J \wedge J\right)
$$

According to [35] and [7] or [17], there is a transport matrix $\Gamma$ which yields the "linear" relation between efforts and flows variables:

$$
f=\Gamma \mathrm{d} e
$$

Onsager's reciprocal relations [35] implies that the transport matrix $\Gamma$ is symmetric, for some special choice for the flows variables (and consequently of their conjugated efforts). As a first approximation, for the $\mathcal{R}$ - field definition, we may consider (as in [7]), that the transport matrix $\Gamma$

$$
\left(\begin{array}{c}
f_{q} \\
\tau \\
J
\end{array}\right)=\underbrace{\left(\begin{array}{ccc}
\star_{\chi} & \cdot & \cdot \\
\cdot & \star_{\kappa} & \cdot \\
\cdot & \cdot & \star_{1 / \eta}
\end{array}\right)}_{\Gamma} \mathrm{d}\left(\begin{array}{c}
T \\
i_{v} \mathbf{v} \\
V_{\text {loop }}
\end{array}\right)
$$

to be diagonal and the diagonal terms $\star_{\chi}, \star_{\kappa}, \star_{1 / \eta}$ to represent respectively the thermal diffusion coefficient, the viscosity, and the electrical resistivity. Vloop is the loop voltage such that the electrical intensity is $E_{\Omega}=\mathrm{d} V_{\text {loop }}$. This constitutive relation (59) completes our model taking into account the irreversible entropy production (dissipation) in the $\mathcal{R}$ - field.

The off-diagonal terms in (61) may play significant roles in the plasma dynamics. Nevertheless, the are until now poorly known and the subject of active researches [17].

\subsubsection{Energy storage element ( $\mathcal{C}$-field)}

The energy storage element in the material domain may be define according to equation (41):

$$
\epsilon=\frac{\star \mathbf{p} \wedge \mathbf{p}}{2}+\mathbf{u}(\mathbf{s}, \mathbf{v})
$$

where the power contributions come from the mechanical domain via kinetic energy $\left(i_{v} \mathbf{v} \wedge \frac{1}{\mathbf{v}} \frac{D \mathbf{p}}{D t}\right)$, from the thermal domain with $\left(T \wedge \frac{D \mathbf{s}}{D t}\right)$ and from the hydraulical domain by $\left(P \wedge \frac{D \mathbf{v}}{D t}\right)$ (see the Gibbs-Duhem equation (46)).

\section{Integration of the complete model with the port-based approach}

Figure 3 presents the complete tokamak $3 D$ model, using the Bondgraph ${ }^{5}$ (cf.[50, Chap.2, p.53]) notations. It includes all energy domains, as well as their

\footnotetext{
${ }^{5}$ Bondgraph is a graphical modelling methodology which applies for "first principle" multidomain dynamical systems. It is based on the explicit representation of the power transfers
} 
inter-domain and multi-domain couplings: electromagnetic, magneto-hydrodynamic, thermal-mechanic and hydrodynamic multi-domain couplings (using StokesDirac and interconnection structures).

The blue part describes the electromagnetic domain (fixed volume balance equations). The Dirac structure $\mathcal{D}_{E M}$ in (12) couples the electric and magnetic domains and stands for the usual Maxwell's equations (13) together with the 0 -junctions (which represents the magnetic and electric densities balance equations). The 1-junction stands for the electrical field balance equation (15) coupling together Lorentz forces (14) and Ohm's law. The electromagnetic energy is accumulated in the energy storage elements $\mathcal{C}:: \mathbb{H}(D)$ and $\mathcal{C}:: \mathbb{H}(B)$ whose constitutive equations are given by (11). It is dissipated by the Joule effect via the $\mathcal{R}$ - field element with a constitutive equation being part of (61). Two usual controlled variables in the electromagnetic domain are "loop voltage" (or total plasma current) from the boundary pair $\left(V_{\text {loop }}, I_{p}\right)$ (generated by the main magnetic coils) and the non-inductive current density from the distributed pair of variables $\left(J_{n i}, \star_{\star} J_{n i}\right)$ (usually generated by antennas).

The red, green, and black parts in the Bondgraph respectively sketch the models for the thermal, mechanical and hydraulic subdomains in the moving frame material domain. The energy storage in the material domain is represented by a field element $\mathcal{C}:: \mathbb{H}=\int_{\mathcal{M}} \frac{\star \mathbf{p} \wedge \mathbf{p}}{2}+\mathbf{u}(\mathbf{s}, \mathbf{v})$ with the internal energy given in the Gibbs-Duhem equation (46). Commonly the ideal gas constitutive equation is used in the material domain since the plasma density is very low. All the balance equations developed in section 4 are realized with the 0 -junctions in the three subdomains. The transport equations (61) and the irreversible entropy source terms (50) are realized in the dissipative $\mathcal{R}$-field which transfers all the dissipated energy to the thermal domain. In each material subdomain, there is a Stokes-Dirac structure (see section 5.2) such as the structure $\mathcal{D}_{2}$ for the hydraulical domain (equation $(56)$ ), $\mathcal{D}_{3}$ for the mechanical domain (equation (57)) and $\mathcal{D}_{4}$ for the thermal domain (equation (58)). In each of these Stokes-Dirac structures, boundary port variables are available for connections with external sensors/actuators systems or more generally with the external environment. Two other used control actions in the material domain, common and important in tokamak applications, are the distributed heating source $S_{\text {heat }}$ in thermal domain and the material injection source $\sigma_{\mathbf{v}}$ in the hydraulical domain.

The electromagnetic volumetric balance equations in the blue part are related to the material domain through the Dirac structure $\mathcal{D}(\mathbf{v})$ defined in (54) and the Eulerian-Lagrangian transformation $\mathcal{T F}:: B=\mathcal{D}_{1}$ as in (51).

This modular multi-physics model allows a separate analysis for (some) interesting subdomain(s) with the appropriate simplifying assumptions. For in-

between energy storage subsystems (i.e. $\mathcal{C}:: \mathbb{H}$ elements), dissipative subsystems (i.e. $\mathcal{R}$ field elements), and power preserving interconnection elements (such as Dirac structures $\mathcal{D}$ or junctions 0 and 1). Power exchanges between the system energy and the environment are "flowing" through boundary or distributed "external" ports). Each power flow in each domain is identified as a pairing between effort and flow variables denoted $e \wedge f$. 
stance, if only the electromagnetic domain is considered and when axisymmetry and quasistatic equilibrium assumptions are made, one obtains the classical 1D resistive diffusion equation commonly used for the control of the poloidal magnetic flux radial profile (see [52] for this reduction and equivalence with the resistive diffusion equation). Besides, the stability of Dirac structures under serial, parallel and feedback interconnection allows guarantees the passivity and allows passivity based control approaches for the interconnected model. 


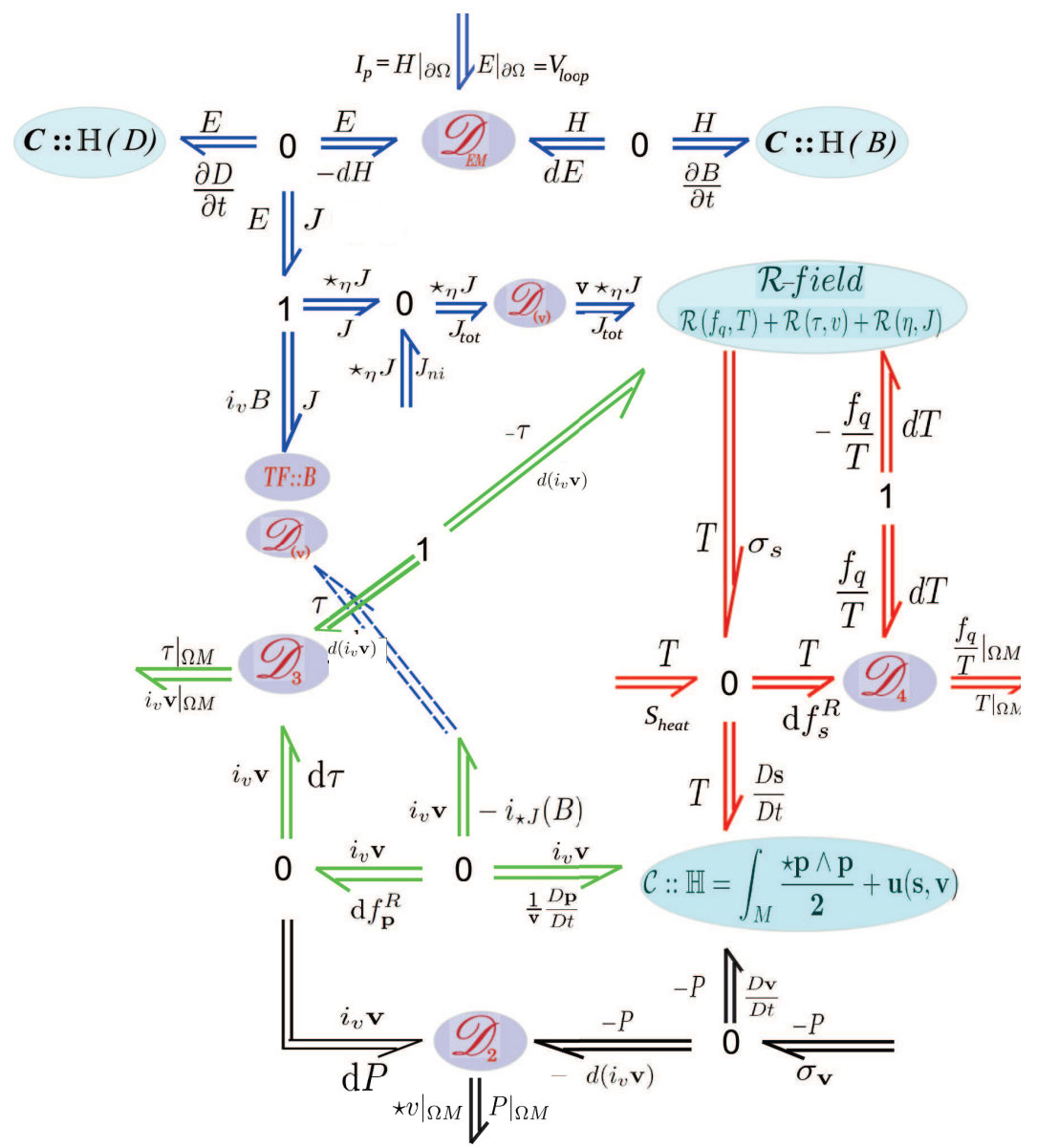

Figure 3: Bondgraph of thermodynamic tokamak system including electromagnetic domain (blue), mechanical domain (green), thermal domain (red) and hydraulic domain (black)

\section{Conclusion}

A complete fluid-like 3D model for the plasma dynamics in tokamaks has been proposed. It is based on the port-Hamiltonian formulation for distributed parameters systems with boundary energy flows. It is built with Stokes-Dirac 
structure and Dirac interconnection structures (including a power preserving MHD coupling structure) on one side and on balance equations or thermodynamics closure relations such as Ohm's law, Lorentz forces or the ideal gas relation, on the other side.

The balance and closure equations in the moving material domain are derived from the Boltzmann equation, using the classical kinetic theory approach. A covariant port-Hamiltonian formulation of these equations is obtained by introducing material derivatives. The entropy balance and the Gibbs-Duhem equations allowed us to compute the irreversible entropy production (source) terms. These entropy production terms and transport phenomena have been used to define TMHD couplings including a transport $\mathcal{R}$ - field (which satisfies Onsager's principle) and Stokes-Dirac interconnection structures.

Now that the global model interconnection structure and constitutive equations are determined, one expects to be able to gradually augment the usual resistive diffusion equation control model (which corresponds only to the electromagnetic balance equations with some restricting assumptions). The first step will be to include a simple thermal model with the heat production from the Joule effect (ohmic resistivity), a simple heat transport diffusion (dispersion) model and the variation of the resistivity with the temperature. This model will allow us to consider the important effect of temperature variations on the resistivity and also to use the non inductive actuators for the heating of the plasma. The second step will be to consider the magneto-hydrodynamic and hydrodynamic part to obtain a control model which includes the bootstrap current effect. Finally, the third step will be the inclusion of the mass balance equation and the supplementary terms and equations related to the fusion reaction.

[1] F.B. Argomedo, C. Prieur, E. Witrant, and S. Brémond. A strict control lyapunov function for a diffusion equation with time-varying distributed coefficients. IEEE Transactions on Automatic Control (2012), 2012.

[2] M. Ariola and A. Pironti. Magnetic Control of Tokamak Plasmas. Advances in Industrial Control. Springer Verlag, London, 2008.

[3] A. Baaiu, F. Couenne, D. Eberard, C. Jallut, Y. Le Gorrec, L. Lefevre, and M. Tayakout-Fayolle. Port-based modelling of mass transfer phenomena. Mathematical and Computer Modelling of Dynamical Systems, 15(3):233$254,2009$.

[4] A. Baaiu, F. Couenne, D. Eberard, C. Jallut, Y. Legorrec, L. Lefèvre, and B. Maschke. Port-based modelling of mass transport phenomena. Mathematical and Computer Modelling of Dynamical Systems, 22(1):1-22, 2009.

[5] A. Baaiu, F. Couenne, L. Lefevre, Y. Le Gorrec, and M. Tayakout-Fayolle. Structure-preserving infinite dimensional model reduction: Application to adsorption processes. Journal of Process Control, 19:394-404, 2009. 
[6] J. Blum. Numerical Simulation and Optimal Control in Plasma Physics. Gauthier-Villars, 1989.

[7] A.H. Boozer. Onsager symmetry of transport in toroidal plasmas. Phys. Fluids, 4(9):2845-2853, September 1992.

[8] M.D. Boyer and E. Schuster. Adaptive nonlinear burn control in tokamak fusion reactors. American Controls Conference, Montréal, Canada (2012), June 2012.

[9] M.D. Boyer and E. Schuster. Nonlinear burn control in tokamak fusion reactors via output feedback. 19th IFAC World Congress (2014), Capetown, South Africa, August 2014.

[10] S.I. Braginskii. Reviews of Plasma Physics, M.A. Leontovich editor (1965).

[11] Y. Choquet-Bruhat and C. De Witt-Morette. Analysis Manifolds and Physics. North-Holland Publ. Co., revised edition, 1982.

[12] T.J. Courant. Dirac manifolds. Trans. American Math. Soc. 319, pages 631-661, 1990.

[13] M. Dalsmo and A.J. van der Schaft. On representations and integrability of mathematical structures in energy-conserving physical systems. SIAM Journal of Control and Optimization, 37(1):54-91, 1999.

[14] S.R. de Groot and P. Mazur. Non-equilibrium thermodynamics. Dover Books on Physics. Dover Publications, 2nd edition edition, 1984.

[15] A. Franco, P. Schott, C. Jallut, and B.M. Maschke. Multi-scale bond graph model of the electrochemical dynamics in a fuel cell. In Proceedings of the of the 5th MathMod conference, Vienna, Autsria, 2006.

[16] T. Frankel. The Geometry of Physics : an Introduction. Cambridge University Press, Cambridge, 2nd edition edition, 2004.

[17] X. Garbet and et al. Thermodynamics of neoclassical and turbulent transport. Plasma Phys. Control. Fusion, (54), April 2012.

[18] O. Gaye, E. Moulay, S. Brémond, L. Autrique, R. Nouailletas, and Y. Orlov. Sliding mode stabilization of the current profile in tokamak plasmas. Conference on Decision and Control (2011), Orlando, Florida, USA, December 2011.

[19] G. Golo, V. Talasila, A.J. van der Schaft, and B. Maschke. Hamiltonian discretization of boundary control systems. Automatica, 40(5):757-771, 2004.

[20] B. Hamroun, L. Lefevre, and E. Mendes. Port-based modelling for open channel irrigation systems. Transactions on Fluid Mechanics, 1(12):9951009, 2006. 
[21] B. Hamroun, L. Lefevre, and E. Mendes. A port-controlled hamiltonian approach to geometric reduction of distributed parameters systems - application to the shallow water equations. International Journal of Numerical Methods in Engineering (2009), 2009.

[22] D.C. Karnopp, D.L. Margolis, and R.C. Rosenberg. System Dynamics: Modeling and Simulation of Mechatronic Systems. John Wiley and Sons, 2006.

[23] A. Kugi. Non-linear Control Based on Physical Models: Electrical, Hydraulic and Mechanical Systems, volume 260 of Lecture Notes in Control and Information Science. Springer, London, Great Britain, 2000.

[24] A. Macchelli and B.M. Maschke. Modeling and Control of Complex Physical Systems - The Port-Hamiltonian Approach, chapter Infinite-dimensional Port-Hamiltonian Systems, pages 211-272. Springer, Sept. 2009. ISBN 978-3-642-03195-3.

[25] A. Macchelli and C. Melchiorri. Modeling and control of the Timoshenko beam. the Distributed Port Hamiltonian approach. SIAM Journal On Control and Optimization, 43(2):743-767, 2004.

[26] J.E. Marsden and A. Weinstein. The Hamiltonian structure of the MaxwellVlasov equations. Physica D: nonlinear phenomena, 4(3):394-406, 1982.

[27] B. Maschke and A.J. van der Schaft. Advanced Topics in Control Systems Theory. Lecture Notes from FAP 2004. Lecture Notes on Control and Information Sciences. Springer, 2005 (115-154).

[28] B.M. Maschke and A. J. van der Schaft. Canonical interdomain coupling in distributed parameter systems: an extension of the symplectic gyrator. In Proc. Int. Mechanical Engineering Congress and Exposition, New- York, USA, Nov. 2001. ASME.

[29] P.J. Morrison and J.M. Greene. Noncanonical Hamiltonian density formulation of hydrodynamics and ideal magnetohydrodynamics. Phys. Rev. Lett., 45(10):790-794, Sep 1980.

[30] P.J. Morrison and J.M. Greene. Hamiltonian and action principle formulations of plasma physics. Physics of Plasmas, 12 (2005), 2005.

[31] G. Nishida and N. Sakamoto. Port-based modeling of magnetohydrodynamics equations for tokamaks. IEEE Control Applications (CCA), pages 842-847, September 2010.

[32] G. Nishida, K. Takagi, and B. Maschke. Multiscale distributed porthamiltonian representation of ionic polymer-metal composite (ipmc). In Proc. IFAC World Congress (2008) 2300-2305. 
[33] G. Nishida, K. Takagi, B. Maschke, and T. Osada. Multi-scale distributed parameter modeling of ionic polymer-metal composite soft actuator. Control Engineering Practice, 19(4):321-334, 2011.

[34] P.J. Olver. Applications of Lie Groups to Differential Equations, volume 107 of Graduate texts in mathematics. Springer, New-York, ii edition, 1993.

[35] L. Onsager. Reciprocal relations in irreversible processes. Phys. Rev. 2265 (38) (1931).

[36] Y. Ou, C. Xu, and E. Schuster. Robust control design for the poloidal magnetic flux profile evolution in the presence of model uncertainties. IEEE Transactions on Plasma Sciences, 32(3):375-382, March 2010.

[37] Y. Ou, C. Xu, E. Schuster, T.C. Luce, J.R. Ferron, M.L. Walker, and D.A. Humphreys. Optimal tracking control of current profile in tokamaks. IEEE Transaction on Control Systems Technology, 19(2):432-441, March 2011.

[38] H. Ouarit, S. Brémond, R. Nouailletas, E. Witrant, and L. Autrique. Validation of plasma current profile model predictive control in tokamaks via simulations. SOFT Porto, Portugal (2010), 2010.

[39] A. Pironti and M. Walker. Fusion, tokamaks and plasma control. IEEE Control Systems Mag., 25:30-43, 2005.

[40] M. Polner and J.J.W. van der Vegt. A hamiltonian vorticity-dilatation formulation of the compressible euler equations. Nonlinear Analysis, (109):113-135, July 2014.

[41] A. Jamiolkowski R.S. Ingarden. Classical Electrodynamics. PWN-Polish Sc. Publ. Elsevier, Warszawa, Poland, 1985.

[42] E. Schuster and M. Krstic. Control of a nonlinear pde system arising from non-burning tokamak plasma transport dynamics. International Journal of Control, 76(11):1116-1124, 2003.

[43] E. Schuster, M. Krstic, and G. Tynan. Nonlinear lyapunov-based burn control in fusion reactor. Fusion Engineering and Design, (63-64):569-575, 2002.

[44] E. Schuster, M. Krstic, and G. Tynan. Burn control in fusion reactors via nonlinear stabilization techniques. Fusion Science and Technology 43 (2003), 2003.

[45] S. Stramigioli. Modeling and IPC Control of Interactive Mechanical Systems: a Coordinate-free Approach, volume 266 of Lect. Notes in Control and Information Sci. Springer, London, Great Britain, 2001.

[46] E. Tassi, P.J. Morrison, F.L. Waelbroeck, and D. Grasso. Hamiltonian formulation and analysis of a collisionless fluid reconnection model. Plasma Physics and Controlled Fusion, 50 (2008), 2008. 
[47] A.J. van der Schaft. $L_{2}$-Gain and Passivity Techniques in Nonlinear Control. Springer Communications and Control Engineering series. SpringerVerlag, London, 2nd revised and enlarged edition, 2000. first edition Lect. Notes in Control and Inf. Sciences, vol. 218, Springer-Verlag, Berlin, 1996.

[48] A.J. van der Schaft and B.M. Maschke. The Hamiltonian formulation of energy conserving physical systems with external ports. Archiv für Elektronik und Übertragungstechnik, 49(5/6):362-371, 1995.

[49] A.J. van der Schaft and B.M. Maschke. Hamiltonian formulation of distributed parameter systems with boundary energy flow. J. of Geometry and Physics, 42:166-174, 2002.

[50] V.Duindam, A. Macchelli, S. Stramigioli, and H. Bruyninckx eds. Modeling and Control of Complex Physical Systems - The Port-Hamiltonian Approach. Springer, Sept. 2009.

[51] Trang.N.M. Vu, L. Lefèvre, R. Nouailletas, and S. Brémond. Geometric discretization for a plasma control model. IFAC Joint conference: 5th Symposium on System Structure and Control (2013); Grenoble, France.

[52] Trang.N.M. Vu, L. Lefèvre, R. Nouailletas, and S. Brémond. Structure preserving reduction for thermo-magneto plasma control model. The 21st International Symposium on Mathematical Theory of Networks and Systems (2014), Groningen, Netherlands, July 07-11 2014.

[53] M.L. Walker, D.A. Humphreys, D. Mazon, D. Moreau, M. Okabayashi, T.H. Osborne, and E. Schuster. Emerging applications in tokamak plasma control. IEEE Control Systems Mag., 26:35-63, 2006.

[54] J. Wesson. Tokamaks. Third edition. Oxford Science Publications, 2004.

[55] E. Witrant, E. Joffrin, S. Brémond, G. Giruzzi, D. Mazon, O. Barana, and P. Moreau. A control-oriented model of the current profile on tokamak plasma. Plasma Physics and Controlled Fusion, 49:1075-1105, 2007. 


\section{AppendixA. Microscopic model (transport equations)}

Using usual tensor notations, the Boltzmann equation for distribution functions $f_{a}(t, r, v)$ for particles of species $a$ with position $r$ and velocity $v$ in the coordinates $\beta$ reads:

$$
\frac{\partial f_{a}}{\partial t}+\frac{\partial}{\partial r_{\beta}}\left(v_{\beta} f_{a}\right)+\frac{\partial}{\partial v_{\beta}}\left(\frac{F_{a \beta}}{m_{a}} f_{a}\right)=C_{a}
$$

For the plasma example in the tokamak we may consider the collisionless case $\left(C_{a}=0\right)$ and external forces $F_{a}=e_{a} E+\frac{e_{a}}{c}[v B]$. We perform then the integration of the products of that equation with $1, m_{a} v_{a}$, and $\frac{m_{a} v_{a}^{2}}{2}$ respectively, over the velocity domain, in order to deduce the mass, momentum, and energy balances (cf. [10, p.208]).

AppendixA.1. Equation of continuity (particle transport equation)

When integrating equation (16) over the velocity domain, we get the particle transport equation:

$$
\begin{aligned}
\int_{\mathbb{R}^{3}}\left(\frac{\partial f_{a}}{\partial t}+\frac{\partial}{\partial r_{\beta}}\left(v_{\beta} f_{a}\right)+\frac{\partial}{\partial v_{\beta}}\left(\frac{F_{a \beta}}{m_{a}} f_{a}\right)\right) d v & =0 \\
\Leftrightarrow \frac{\partial n_{a}}{\partial t}+\frac{\partial}{\partial r_{\beta}}\left(n_{a} \bar{v}_{a}\right) & =0
\end{aligned}
$$

where $a=e$ or $i$. In this last expression one assumes that the third term of (16) vanishes rapidly as $v \rightarrow \infty$ and the resulting term in the integration may be neglected when compared to the two first terms. Omitting the species subscripts, one gets:

$$
\frac{\partial n}{\partial t}+\nabla \cdot(n \bar{v})=0
$$

Using the usual material derivative:

$$
\frac{d}{d t}=\frac{\partial}{\partial t}+\bar{v} \nabla
$$

one gets:

$$
\begin{aligned}
\frac{d n}{d t} & =\frac{\partial n}{\partial t}+\bar{v} \nabla \cdot n \\
& =-\nabla \cdot(n \bar{v})+\bar{v} \nabla \cdot n \\
& =-n \nabla \cdot \bar{v}-\bar{v} \nabla n+\bar{v} \nabla \cdot n \\
& =-n \nabla \cdot \bar{v}
\end{aligned}
$$

as given in equation (20). 


\section{AppendixA.2. Equation of motion (momentum transport)}

From now, we denote respectively $m, n, v$ the corresponding quantities $m_{a}, n, v_{a}$ for the species $a$. Multiplying the Boltzmann (16) by $m v$, and integrating over the velocity domain, one gets:

$$
\begin{aligned}
m \int_{\mathbb{R}^{3}} v\left(\frac{\partial f_{a}}{\partial t}+\frac{\partial}{\partial r_{\beta}}\left(v_{\beta} f_{a}\right)+\frac{\partial}{\partial v_{\beta}}\left(\frac{F_{a \beta}}{m_{a}} f_{a}\right)\right) d v=0 \\
\Leftrightarrow \frac{\partial(m n \bar{v})}{\partial t}+\nabla \cdot\left(m n\left\langle v v_{\beta}\right\rangle\right)-e_{a} n\left(E_{a}+\frac{1}{c}[\bar{v} B]_{a}\right)=0
\end{aligned}
$$

with $e_{a}=e_{e}=-e$ for electrons. Decomposing the velocity field $v=\bar{v}+v^{\prime}$, with the mean velocity $\bar{v}$ and a random velocity $v^{\prime}$ whose $\left\langle v^{\prime}\right\rangle=0$, then:

$$
\left\langle v v_{\beta}\right\rangle=\overline{v v}_{\beta}+\left\langle v^{\prime} v_{\beta}^{\prime}\right\rangle
$$

and the second term of (A.3) is:

$$
\begin{array}{r}
\nabla \cdot\left(m n\left\langle v v_{\beta}\right\rangle\right)=\nabla \cdot\left(m n \bar{v}_{\beta}\right)+\nabla \cdot\left(m n\left\langle v^{\prime} v_{\beta}^{\prime}\right\rangle\right) \\
=\quad m n \bar{v}_{\beta} \nabla \cdot(\bar{v})+m \bar{v} \nabla \cdot\left(n \bar{v}_{\beta}\right)+ \\
+\nabla \cdot\left(m n\left\langle v^{\prime} v_{\beta}^{\prime}\right\rangle\right)
\end{array}
$$

One obtains then the pressure tensor in the form:

$$
\mathbf{P}_{a \beta}=\int_{\mathbb{R}^{3}} m v^{\prime} v_{\beta}^{\prime} f d v=m n\left\langle v^{\prime} v_{\beta}^{\prime}\right\rangle=P \delta_{a \beta}+\tau_{a \beta}
$$

where $P$ denotes the scalar pressure and $\tau$ the stress tensor, whereas:

$$
\left\{\begin{array}{l}
P=n m\left\langle v^{\prime 2}\right\rangle / 3=n T \\
\tau_{a \beta}=n m\left\langle v^{\prime} v_{\beta}^{\prime}-\left(v^{\prime 2} / 3\right) \delta_{a \beta}\right\rangle
\end{array}\right.
$$

On the other hand, thanks to the continuity equation in (A.3), the first term of equation (A.6) is derived:

$$
\begin{aligned}
\frac{\partial\left(m n \bar{v}_{a}\right)}{\partial t} & =m n \frac{\partial \bar{v}}{\partial t}+m \bar{v} \frac{\partial n}{\partial t} \\
& =m n \frac{\partial \bar{v}}{\partial t}-m \bar{v}\left[\nabla \cdot\left(n \bar{v}_{\beta}\right)\right]
\end{aligned}
$$

Then from (A.15-A.11), and (A.4) the momentum transport can be rewritten as:

$$
n m \frac{d \bar{v}}{d t}=-\nabla P-\nabla \times \tau+e n\left(E_{a}+\frac{1}{c}[\bar{v} B]_{a}\right)
$$




\section{AppendixA.3. Energy transport}

Multiplying the Boltzmann equation (16) by $\frac{m v^{2}}{2}$ and integration over the velocity domain, one gets:

$$
\begin{aligned}
\int_{\mathbb{R}^{3}} \frac{m v^{2}}{2}\left(\frac{\partial f_{a}}{\partial t}+\frac{\partial}{\partial x_{\beta}}\left(v_{\beta} f_{a}\right)+\frac{\partial}{\partial v_{\beta}}\left(\frac{F_{a \beta}}{m_{a}} f_{a}\right)\right) d v & =0 \\
\Leftrightarrow \frac{\partial}{\partial t}\left(\frac{m n}{2}\left\langle v^{2}\right\rangle\right)+\nabla \cdot\left(\frac{m n}{2}\left\langle v^{2} v_{\beta}\right\rangle\right)-e n(E \bar{v}) & =0
\end{aligned}
$$

In first term, the total energy $\epsilon$ (which includes the kinetic energy) may be written with the help of (A.10):

$$
\begin{aligned}
\epsilon=\frac{m n}{2}\left\langle v^{2}\right\rangle & =\frac{m n}{2} \bar{v}^{2}+\frac{m n}{2}\left\langle v^{\prime 2}\right\rangle \\
& =\quad \frac{m n}{2} \bar{v}^{2}+\frac{3}{2} n T
\end{aligned}
$$

The second term in (A.13) corresponds to the divergence of the energy flux:

$$
\begin{aligned}
\left\langle v^{2} v_{\beta}\right\rangle & =\left\langle\left(\bar{v}+v^{\prime}\right)^{2}\left(\bar{v}_{\beta}+v_{\beta}^{\prime}\right)\right\rangle \\
& =\bar{v}^{2} \bar{v}_{\beta}+\left\langle v^{\prime 2}\right\rangle \bar{v}_{\beta}+2 \bar{v}\left\langle v^{\prime} v_{\beta}^{\prime}\right\rangle+\left\langle\left(v^{\prime}\right)^{2} v_{\beta}^{\prime}\right\rangle \\
& =\bar{v}^{2} \bar{v}_{\beta}+\frac{(3 n T) \bar{v}_{\beta}}{n m}+\frac{2(\tau+n T) \bar{v}}{n m}+\frac{2 \mathbf{q}}{n m}
\end{aligned}
$$

Hence thanks to the definition of the pressure $P$ and the stress tensor $\tau$ in (A.10), using the flux density of heat:

$$
\mathbf{q}=\int_{\mathbb{R}^{3}} \frac{m}{2} v^{\prime 2} v f d v=m n\left\langle\frac{v^{\prime 2}}{2} v\right\rangle
$$

the energy balance equation in (A.13) may be written:

$$
\begin{array}{r}
\frac{\partial}{\partial t}\left(\frac{m n}{2} \bar{v}^{2}+\frac{3}{2} n T\right)+\nabla \cdot\left\{\left(\frac{m n}{2} \bar{v}^{2}+\frac{5}{2} n T\right) \begin{array}{r}
\left.\bar{v}_{\beta}+\tau \bar{v}+\mathbf{q}\right\} \\
-e n(E \bar{v})=0
\end{array}\right.
\end{array}
$$

Using the material derivative (A.4) one gets:

$$
\begin{aligned}
\frac{d \epsilon}{d t} & =\frac{\partial \epsilon}{\partial t}+\nabla \cdot\left(\epsilon \bar{v}_{\beta}\right) \\
& =-\nabla \cdot\left[(\epsilon+n T) \bar{v}_{\beta}+\tau \bar{v}+\mathbf{q}\right]+e n(E \bar{v})+\nabla \cdot\left(\epsilon \bar{v}_{\beta}\right) \\
& =-\nabla \cdot\left[n T \bar{v}_{\beta}+\tau \bar{v}+\mathbf{q}\right]+e n(E \bar{v})
\end{aligned}
$$




\section{AppendixA.4. Equation of internal energy and entropy equation}

The internal energy balance may be derived by using the equations of continuity and motion here above to eliminate the kinetic energy in equation (A.16). We first remind the kinetic rate term:

$$
\begin{aligned}
\frac{d}{d t}\left(\frac{m n}{2} \bar{v}^{2}\right)= & \left(\frac{m \bar{v}^{2}}{2}\right) \frac{d}{d t} n+m n \bar{v} \frac{d}{d t}(\bar{v}) \\
= & -\left(\frac{m \bar{v}^{2}}{2}\right) \nabla \cdot \bar{v}+ \\
& +\bar{v}\left(-\nabla P-\nabla \times \tau+e n\left(E+\frac{1}{c}[\bar{v} B]\right)\right) \\
= & -\frac{3}{2} n T \nabla \cdot \bar{v}+\bar{v}(-\nabla P-\nabla \times \tau+e n E)
\end{aligned}
$$

due to (A.5) and (A.12), and the fact that $\bar{v}[\bar{v} B]=0$. Then (A.17) becomes:

$$
\begin{aligned}
\frac{d}{d t}\left(\frac{m n}{2} \bar{v}^{2}\right)+\frac{d}{d t}\left(\frac{3}{2} n T\right) & =-\nabla \cdot[P \bar{v}+\tau \bar{v}+\mathbf{q}]+e n(E \bar{v}) \\
\Leftrightarrow-\frac{3}{2} n T \nabla \cdot \bar{v}+\frac{d}{d t}\left(\frac{3}{2} n T\right) & =-P \nabla \cdot \bar{v}-\tau \nabla \cdot \bar{v}-\nabla \cdot \mathbf{q} \\
\Leftrightarrow \frac{3}{2} n \frac{d}{d t} T \quad & =-P \nabla \cdot \bar{v}-\tau \nabla \cdot \bar{v}-\nabla \cdot \mathbf{q}
\end{aligned}
$$

Finally, the internal energy equation is deduced:

$$
\frac{3}{2} n \frac{d}{d t} T=-P \nabla \cdot \bar{v}-\tau \nabla \cdot \bar{v}-\nabla \cdot \mathbf{q}
$$

